\title{
A Monte Carlo adapted finite element method for dislocation simulation of faults with uncertain geometry
}

\author{
P ZaKian, N KhaJi* and M Soltani \\ Department of Civil and Environmental Engineering, Tarbiat Modares University, P.O. Box 14115-397, \\ Tehran, Iran. \\ *Corresponding author. e-mail: nkhaji@modares.ac.ir
}

MS received 16 April 2016; revised 15 March 2017; accepted 10 May 2017; published online 10 October 2017

Dislocation modelling of an earthquake fault is of great importance due to the fact that ground surface response may be predicted by the model. However, geological features of a fault cannot be measured exactly, and therefore these features and data involve uncertainties. This paper presents a Monte Carlo based random model of faults with finite element method incorporating split node technique to impose the effects of discontinuities. Length and orientation of the fault are selected as random parameters in the domain model, and hence geometrical uncertainties are encountered. Mean and standard deviation values, as well as probability density function of ground surface responses due to the dislocation are computed. Based on analytical and numerical calculation of dislocation, two approaches of Monte Carlo simulations are proposed. Various comparisons are examined to illustrate the capability of both methods for random simulation of faults.

Keywords. Monte Carlo simulation; stochastic modeling; split node technique; finite element method; earthquake fault dislocation.

\section{Introduction}

In material science, a crystallographic defect is a dislocation (or irregularity) within a crystal structure. Similarly, the slip of an active fault may be considered as a kind of dislocation. The theory which describes the elastic field of defects was originally presented by Volterra (1907), when the mathematics of the dislocation theory was formed. This theory concerns the state of self-stress in a body which is discontinuously deformed. A dislocation is thought as a two-dimensional (2D) manifold, along which the material has been cut, displaced, welded together, and released, subsequently. It is supposed that the considered system experiences a new minimum energy state of self-stressed equilibrium. The magnitude and direction of the displacement jump (or slip) is a vector field over the dislocation plane, where a slip vector has normal components, the material opens, and is added to fill the void, just as it happens when a dyke intrudes a fault (van Zwieten et al. 2013). The displacements are discontinuous at the dislocation plane and continuous elsewhere.

There are several approaches to model elastic dislocation of an active fault (van Zwieten et al. 2013). Analytical methods are rapid and accurate, but are often restricted to simple problems without complexity. On the other hand, numerical methods are usually utilized for complex cases with some improvements for handling 
discontinuous media (Manglik et al. 2008). Split node technique (SNT) was first introduced by Melosh and Raefsky (1981). This method may be implemented in finite element method (FEM) to consider the discontinuity effects of fault without direct modeling of the discontinuity. Even though the SNT was proposed in 1981, this idea is still a robust tool as it can handle anisotropy, heterogeneity, topography, distributed slip and curved fault effects (Melosh and Raefsky 1983; Melosh and Williams 1989; Andrews 1999; Dalguer and Day 2006, 2007; van Zwieten et al. 2013). Recently, van Zwieten et al. (2014) developed the weaklyenforced slip method to model tectonic faults without explicitly simulating discontinuities.

Uncertainty always exists in scientific measurements due to human and instrumental defects. These errors become larger when geologic data is the target, because accurate measurement of geologic data is difficult. Therefore, reliability analysis of these systems becomes a crucial subject. A few investigations are focused on stochastic modeling of fault dislocations. Kagan (1982) incorporated the time-magnitude model of earthquake occurrence developed earlier, and then simulated synthetic sequences by the Monte Carlo method, to reproduce all known magnitude-space-time statistical properties of real earthquake catalogues. In order to testify the applicability of statistical models, Toki et al. (1987) proposed a Monte Carlo based finite element model enriched by joint element for fault rupture process to predict near field ground motion under uncertain stress and strength distribution. Hori (2011) and Hori et al. (2003) employed stochastic finite element method and bounding medium analysis for incorporating material uncertainty in earthquake wave propagation and surface fault formation. Hainzl et al. (2013) accomplished a systematic comparison with deterministic simulations based on rate- and state-dependent friction, high-resolution representations of fault systems, and quasi-dynamic rupture propagation.

In this paper, a Monte Carlo simulation (MCS) based random model of faults using the FEM is presented. For this purpose, the SNT is incorporated to take into account the effects of discontinuities. Based on the best knowledge of the authors, this is the first application of the SNT in random dislocation using the FEM, particularly for the sake of considering geometric uncertainty. Here, the undertaken problem is fault dislocation with random geometry so that the length of the fault is selected as a random variable. Dislocation modeling of an active fault is remarkable, as ground surface response may be predicted by this kind of simulations. In this paper, two approaches of the MCS are presented based on analytical and numerical solution of dislocation. Numerical solution is handled by a split node technique based finite element method (SNT-FEM), while analytical one is carried out using available closed-form formulations for ground surface response due to dislocation.

\section{Elastic dislocation of an earthquake fault}

\subsection{Analytical solution of dislocation}

Closed-form solutions of fault dislocations were proposed by some researchers (see for example Okada 1992; Rani and Singh 1992; Rani and Bala 2013 and others). Static deformation of a uniform half-space due to a long dip-slip fault (as shown in figure 1) is solved in this section. The displacement of ground surface subject to fault slip of $b$, in $x$-direction is given by

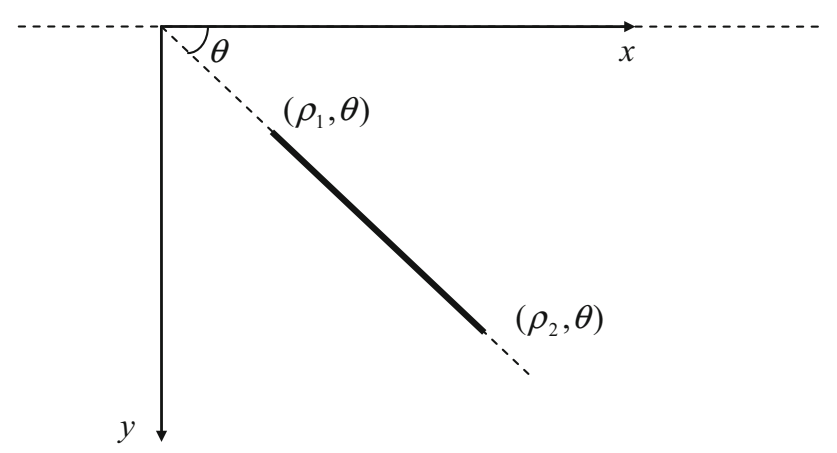

Figure 1. Configuration of a long dip-slip fault in 2D halfspace, where $\left(\rho_{1}, \theta\right)$ and $\left(\rho_{2}, \theta\right)$ denote the top and bottom ends of the fault in polar coordinates.

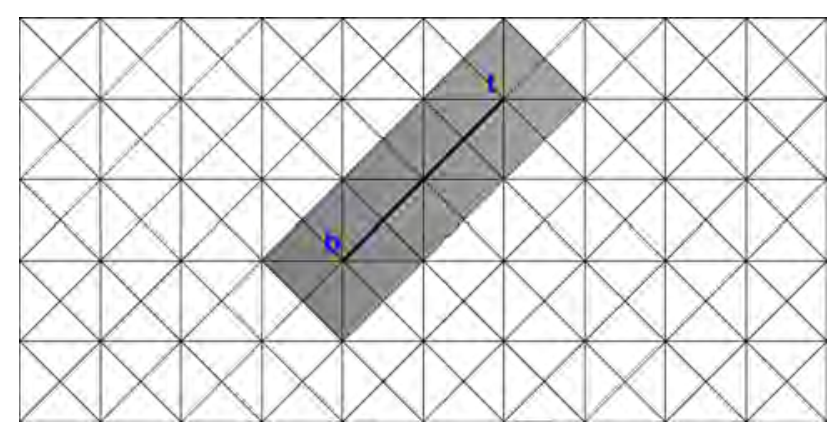

Figure 2. A typical triangular mesh for a domain comprising a fault $(\mathrm{t}-\mathrm{b})$, in which the elements contributing in the SNT are highlighted by grey colour. 


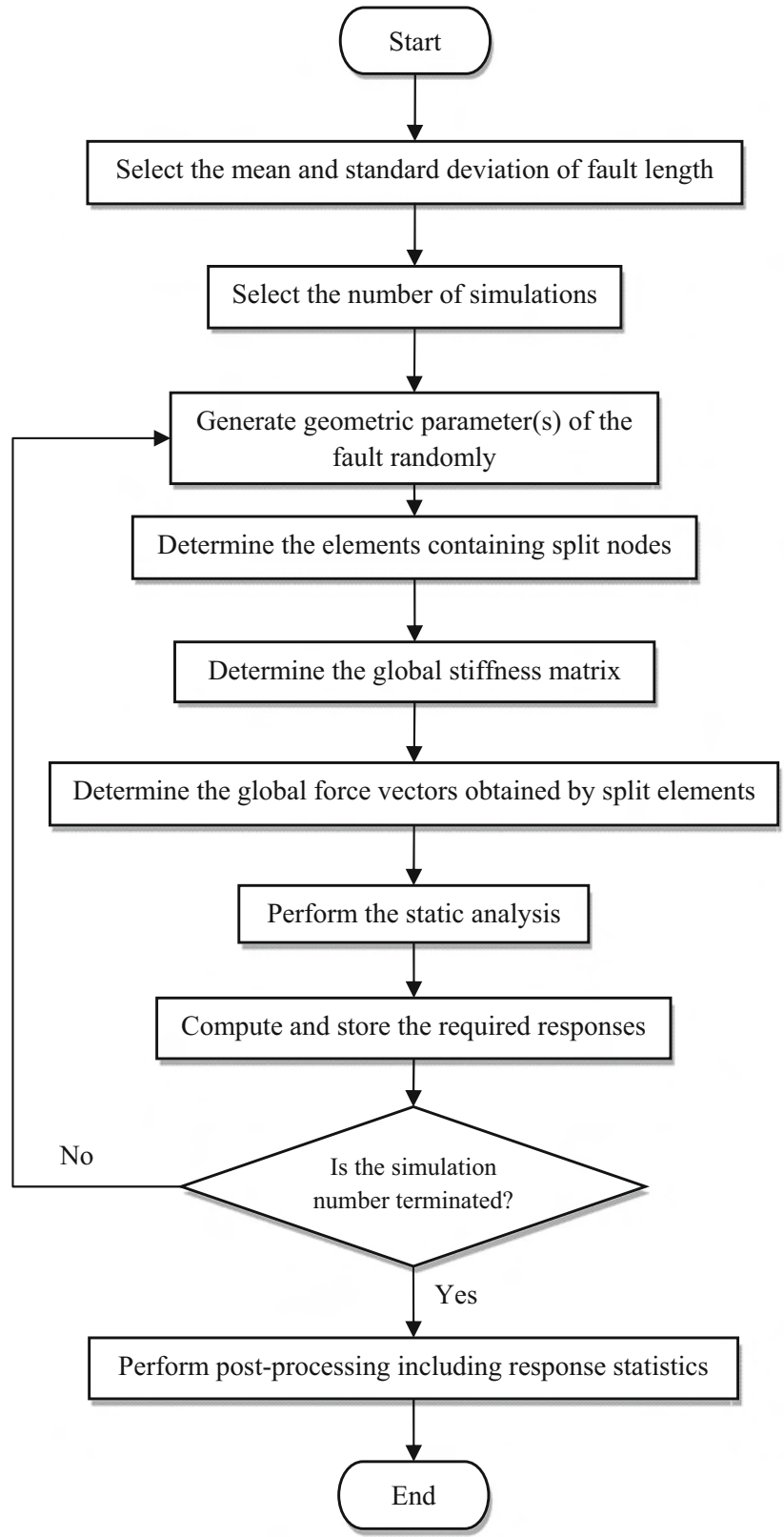

Figure 3. Flowchart of the MCS-based SNT-FEM.

$$
\begin{aligned}
U_{x}= & \frac{b}{2 \pi}\left\{\frac{1-2 \nu}{2(1-\nu)} \sin \theta \ln \left(\frac{R_{1}}{R_{2}}\right)\right. \\
& +\cos \theta \tan ^{-1}\left(\frac{x-\rho \cos \theta}{y+\rho \sin \theta}\right) \\
& -\cos \theta \tan ^{-1}\left(\frac{x-\rho \cos \theta}{y-\rho \sin \theta}\right) \\
& -\frac{(x \sin \theta-y \cos \theta)(x-\rho \cos \theta)}{2(1-\nu)} \\
& \times\left(\frac{1}{R_{1}^{2}}-\frac{1}{R_{2}^{2}}\right)
\end{aligned}
$$

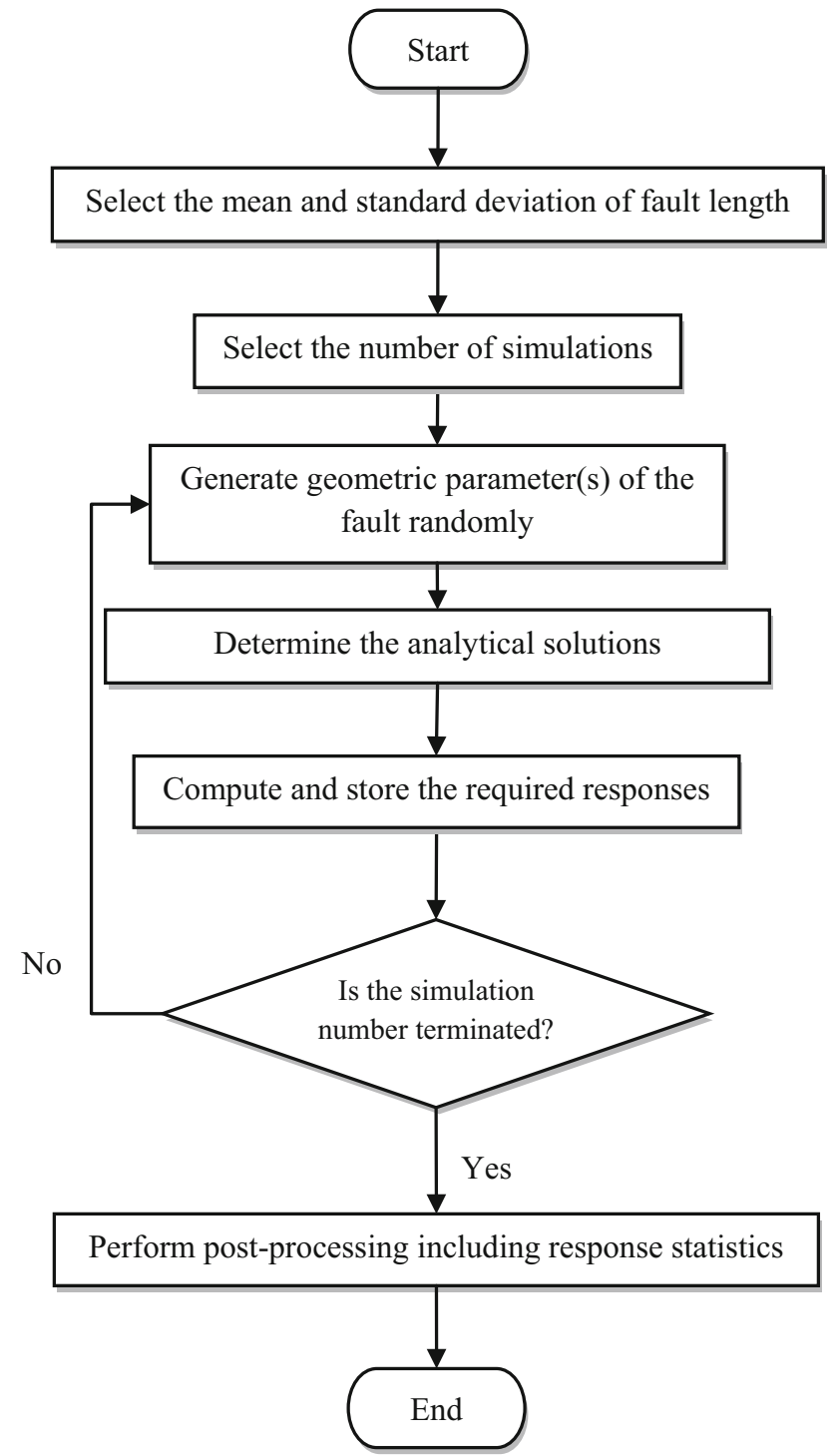

Figure 4. Flowchart of the MCS-based AS.

$$
\begin{aligned}
& +\frac{2 \rho \sin \theta}{R_{2}^{2}}\left(\frac{1-2 \nu}{2(1-\nu)} y \sin \theta+s-x \cos \theta\right) \\
& \left.+\frac{2 y \sin \theta(x \sin \theta+y \cos \theta)}{1-\nu} \frac{\rho(x-\rho \cos \theta)}{R_{2}^{4}}\right\}\left.\right|_{\rho_{1}} ^{\rho_{2}}
\end{aligned}
$$

Similarly, the displacement of ground surface in $y$-direction is

$$
\begin{aligned}
U_{y}= & \frac{b}{2 \pi}\left\{-\frac{1-2 \nu}{2(1-\nu)} \cos \theta \ln \left(\frac{R_{1}}{R_{2}}\right)\right. \\
& -\sin \theta \tan ^{-1}\left(\frac{y+\rho \sin \theta}{x-\rho \cos \theta}\right) \\
& +\sin \theta \tan ^{-1}\left(\frac{y-\rho \sin \theta}{x-\rho \cos \theta}\right)
\end{aligned}
$$




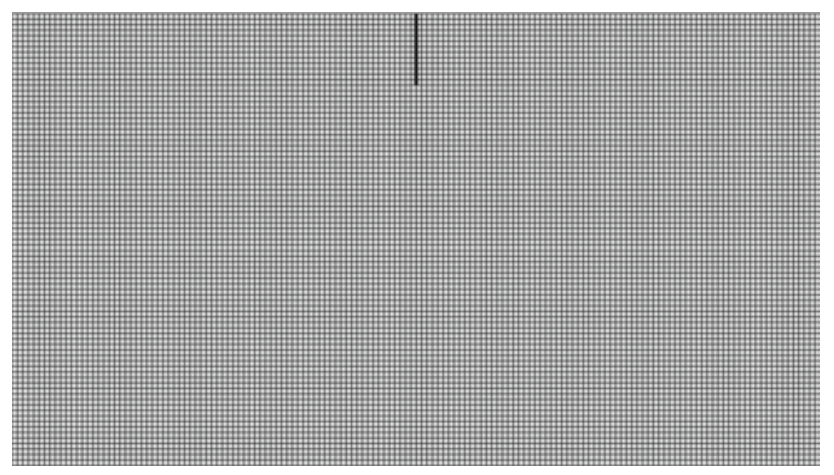

$$
\begin{aligned}
& +\frac{\rho \sin \theta}{R_{2}^{2}}(2(x \sin \theta+y \cos \theta) \\
& \left.-\frac{1}{1-\nu}(x \sin \theta+2 y \cos \theta)\right) \\
& -\frac{1}{2(1-\nu)}(x \sin \theta-y \cos \theta) \\
& \times\left(\frac{(y-\rho \sin \theta)}{R_{1}^{2}}-\frac{(y+\rho \sin \theta)}{R_{2}^{2}}\right) \\
& \left.+\frac{2 y \sin \theta(x \sin \theta+y \cos \theta)}{1-\nu} \frac{\rho(y+\rho \sin \theta)}{R_{2}^{4}}\right\}\left.\right|_{\rho_{1}} ^{\rho_{2}}
\end{aligned}
$$

Figure 5. The vertical outcropped fault shown by black solid line, and the finite element mesh of the domain.
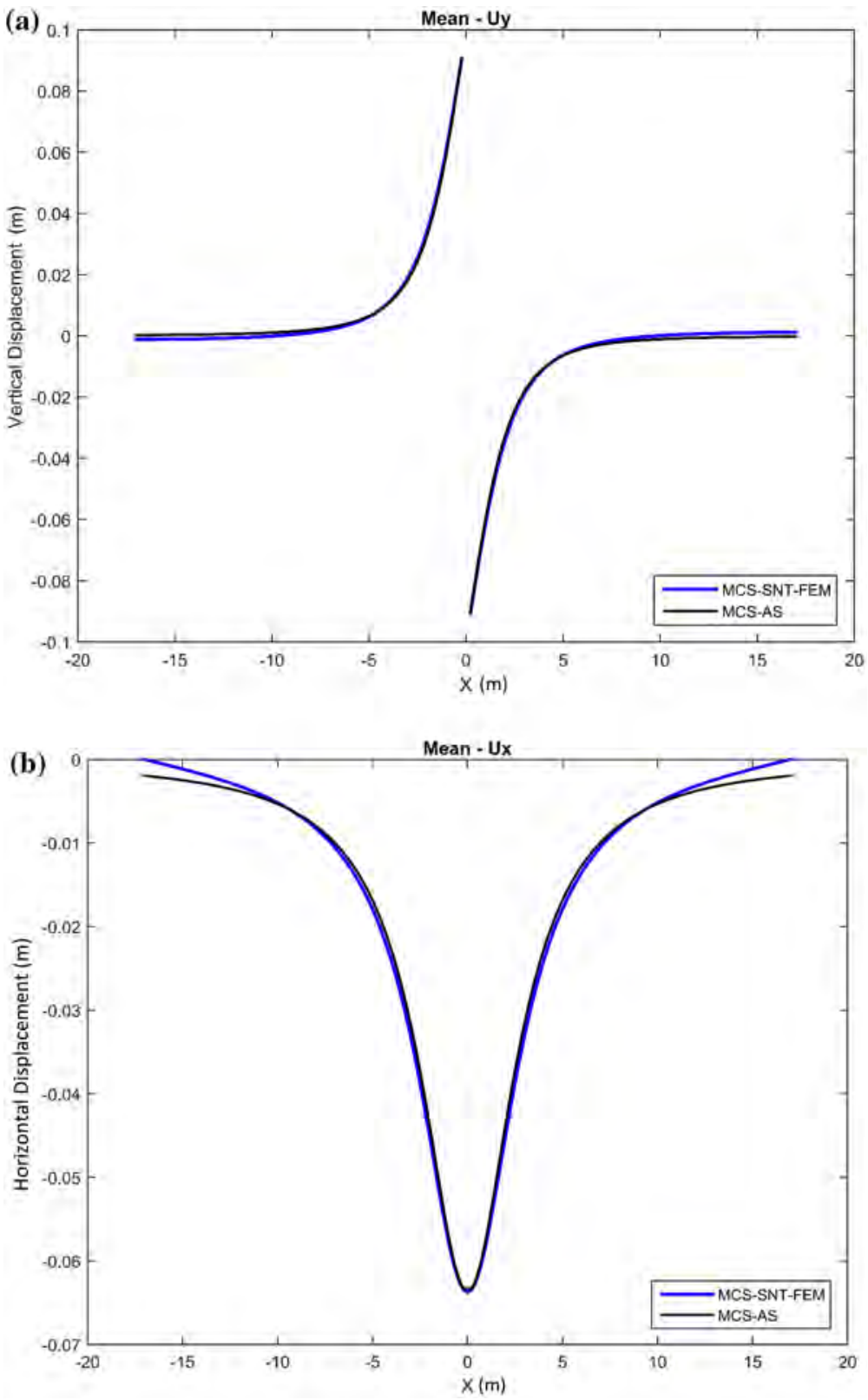

Figure 6. Response mean values of ground surface: (a) vertical displacement, and (b) horizontal displacement. 
where $\nu$ represents Poisson's ratio, $\theta$ indicates the dip angle, and $\rho_{1} \leq \rho \leq \rho_{2}$. In addition, $(x, y)$ is the coordinate of the receiver location. Furthermore, $R_{1}$ and $R_{2}$ are defined as

$$
\begin{aligned}
& R_{1}=\sqrt{(x-\rho \cos \theta)^{2}+(y-\rho \sin \theta)^{2}}, \\
& R_{2}=\sqrt{(x-\rho \cos \theta)^{2}+(y+\rho \sin \theta)^{2}} .
\end{aligned}
$$

Also, $x_{e}(=\rho \cos \theta)$ and $y_{e}(=\rho \sin \theta)$ denote the coordinates of line source location. The length of the fault is indicated by $L=\rho_{2}-\rho_{1}$.

\subsection{Numerical solution of dislocation with the SNT-FEM}

For complex domains, the problem may not be solved by analytical methods. In this situation, a suitable numerical method is an alternative to solve the problem. FEM is an appropriate choice for analysis of dislocation when enriched by the SNT. The FEM has the ability to solve the problem defined for any given conditions including topography, anisotropy, and heterogeneity effects.

If the finite element mesh is constructed to be compatible with the fault (i.e., the dislocation
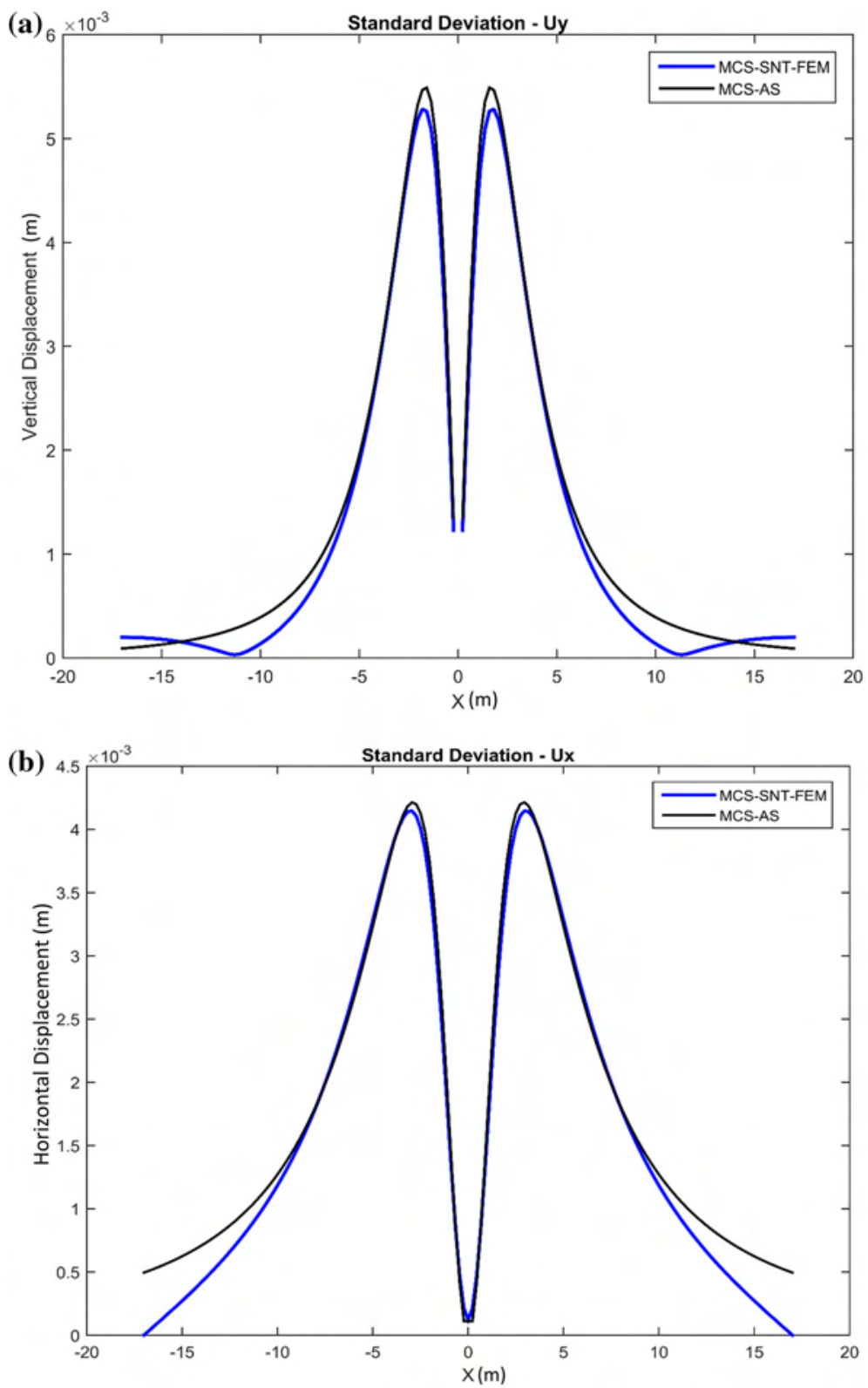

Figure 7. Response standard deviation values of ground surface: (a) vertical displacement, and (b) horizontal displacement. 
coincides with finite element edges), then one may reach to the concept of the SNT (Melosh and Raefsky 1981). Elegance and efficiency are what make the SNT popular and desirable. In the first step of implementing the SNT into the FEM, one should determine the elements whose nodes are connected to the fault. As already mentioned, this method requires a compatible mesh so that the fault aligns with the edges of the contributing elements. A typical FEM mesh for analysis of a fault is depicted in figure 2, in which the split node contributing elements are marked by grey colour. If total slip magnitude is considered as $b$, then the slip of each side of the fault is assumed as $\frac{1}{2} b$ with opposite signs. The contributing elements of right and left sides of the fault have the following force vectors

$$
\begin{aligned}
& \mathbf{f}^{e+}=\mathbf{k}^{e+} \boldsymbol{\delta}^{+}, \\
& \mathbf{f}^{e-}=\mathbf{k}^{e-} \boldsymbol{\delta}^{-},
\end{aligned}
$$

where $\boldsymbol{\delta}^{+}$implies the displacement vector of a contributing element at right side of the fault. This vector is populated by zero entries, except for the nodes connected to the fault, which are replaced by $\frac{1}{2} b \cos \alpha$ and $\frac{1}{2} b \sin \alpha$ entries, for $x$ and $y$ degrees of freedom (DOFs), respectively. The displacement vector of left side $\delta^{-}$is defined as the same as the right side, but non-zero entries are $-\frac{1}{2} b \cos \alpha$ and $-\frac{1}{2} b \sin \alpha$, for $x$ and $y$ DOFs, respectively. The fault is oriented through angle $\alpha$ with respect to horizontal line. In addition, $\mathbf{f}^{e+}$ and $\mathbf{f}^{e-}$ denote right and left side force vectors, respectively; and $\mathbf{k}^{e}$ is the element stiffness matrix. Clearly, only the contributing elements' forces have non-zero terms, and hence force vectors of other elements include zero terms. All force vectors should obviously be assembled for final calculation. Element stiffness matrices remained unchanged and their assemblage is ordinarily performed. Later, by having global stiffness matrix and force vector, the well-known equation of linear static analysis is assembled and is given as:

$$
\mathbf{F}=\mathbf{K} \boldsymbol{\Delta},
$$

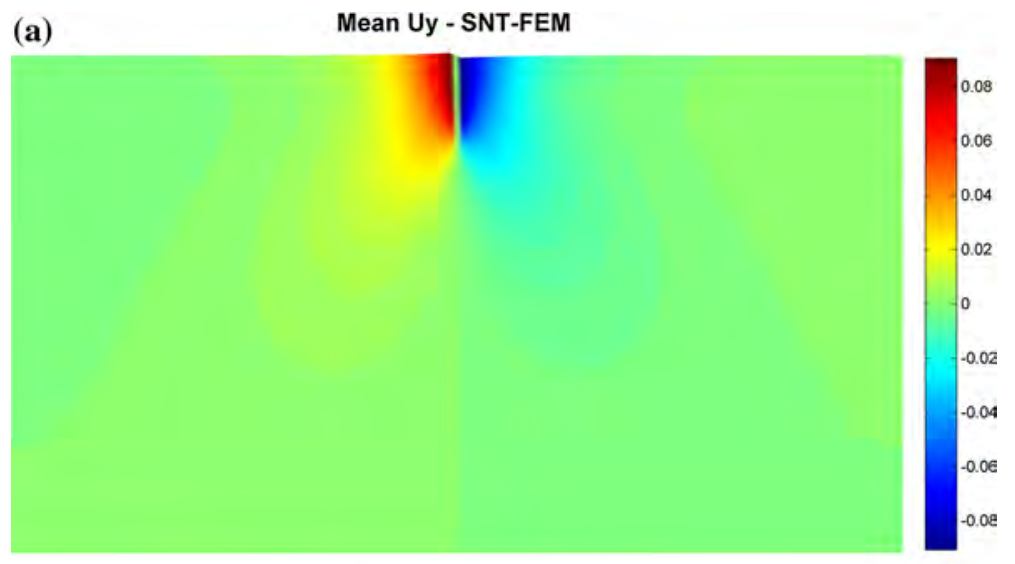

(b) Mean Ux - SNT-FEM

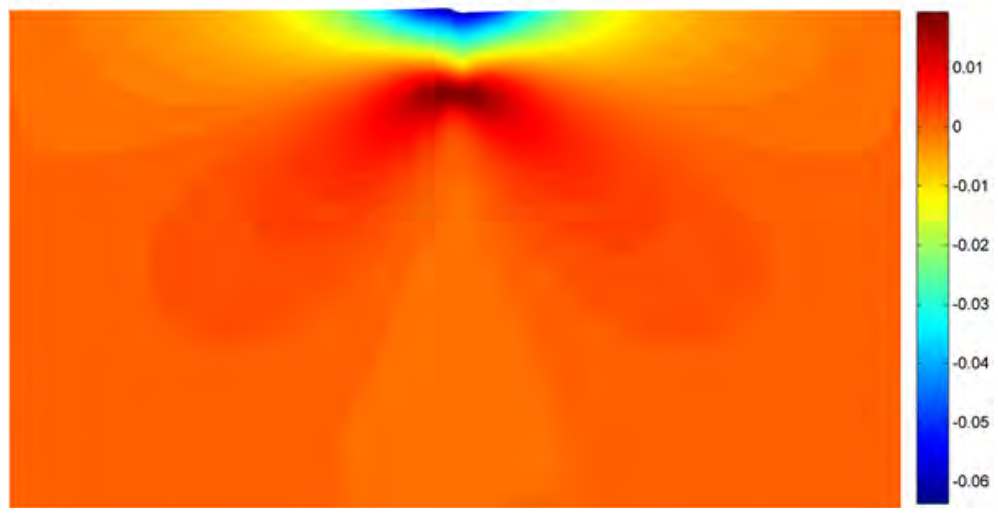

Figure 8. Displacement field contours of mean values in the domain: (a) vertical displacement and (b) horizontal displacement (in $\mathrm{m}$ ). 
in which $\mathbf{K}, \mathbf{F}$ and $\boldsymbol{\Delta}$ are global stiffness matrix, global force and displacement vectors, respectively. Whenever $\boldsymbol{\Delta}$ is gained, the surface responses are determined using the nodal displacements at $x$ and $y$ directions. However, the displacement of nodes locating on the fault should sometimes be broken by compelling the FEM responses, as the SNT-FEM method is an inherently continuous approach.

\section{Monte Carlo-based approaches for elastic dislocation}

Two approaches are proposed in this study: (1) MCS-based on analytical solution (AS), and (2) MCS-based on the SNT-FEM. The MCS is a robust method for probabilistic analysis of engineering systems that can deal with linear and nonlinear analysis as well as Gaussian and non-Gaussian random fields. Additionally, the MCS is a versatile benchmark for assessment and validation of other methods of reliability analysis. In this study, all developed programs are coded in MATLAB environment.

\subsection{MCS-based SNT-FEM}

In this subsection, the MCS-based SNT-FEM is presented, in which three main problems should be resolved. Firstly, since the length of the fault is randomly generated at each simulation, the coded program should select the split nodes and the corresponding elements automatically. Secondly, the generated length of the fault is not always an integer coefficient of element size. And thirdly, the split elements' forces and stiffness matrices should be calculated at each simulation which takes significant computing time. These three problems increase the complexity of the programming.

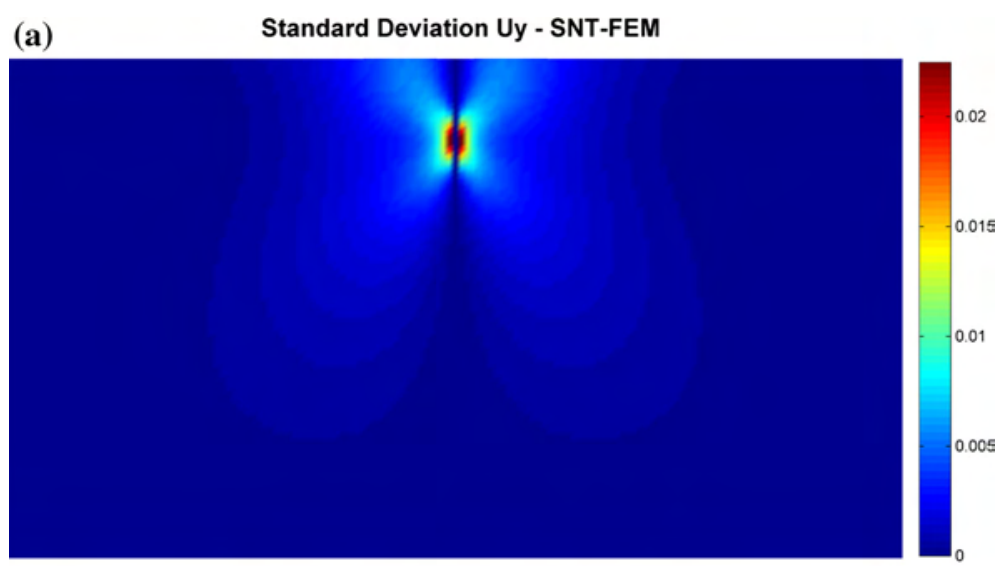

(b)

Standard Deviation Ux - SNT-FEM

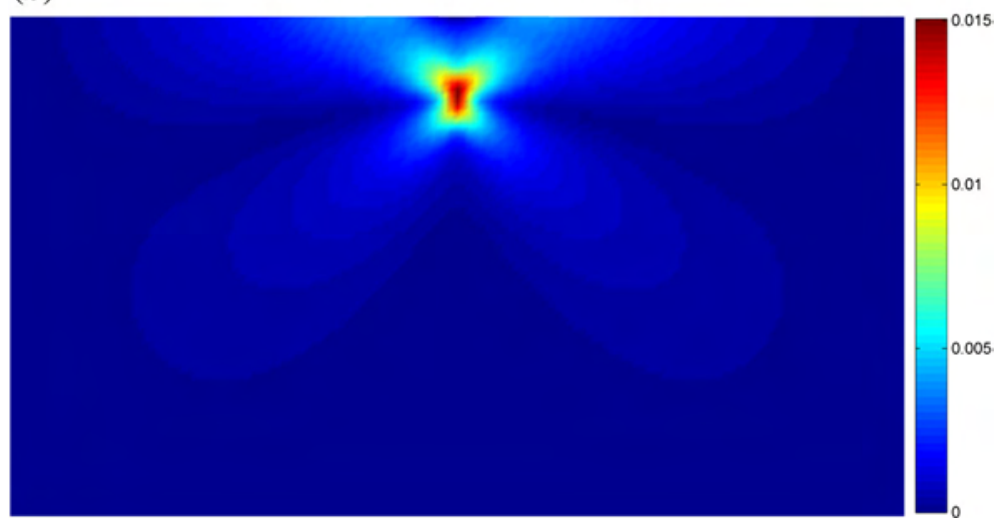

Figure 9. Displacement field contours of standard deviation values in the domain: (a) vertical displacement and (b) horizontal displacement (in $\mathrm{m}$ ). 
Here, some treatments are given to alleviate these difficulties. For the firstone, a computer function is coded to find the split nodes and the corresponding contributed elements. For the second one, two ways may be implemented as follows: (a) Using an adaptive mesh generator at each simulation such that the length of the fault is always an integer coefficient of element size; (b) Using a fine mesh such that one can solve the problem with desirable accuracy, and then round the randomly generated fault length so that the length of the fault becomes an integer coefficient of element size. The last way is employed herein as it is much efficient computationally. For the third one, a fine mesh is selected for all simulations as global stiffness matrix is not changed in the SNT procedure, whereby it can be calculated once before the MCS procedure. Furthermore, force vectors of likely (or, potential) split elements may be computed and stored in this stage as well. These computational tricks enhance the efficiency of the proposed method. Essential steps of the proposed
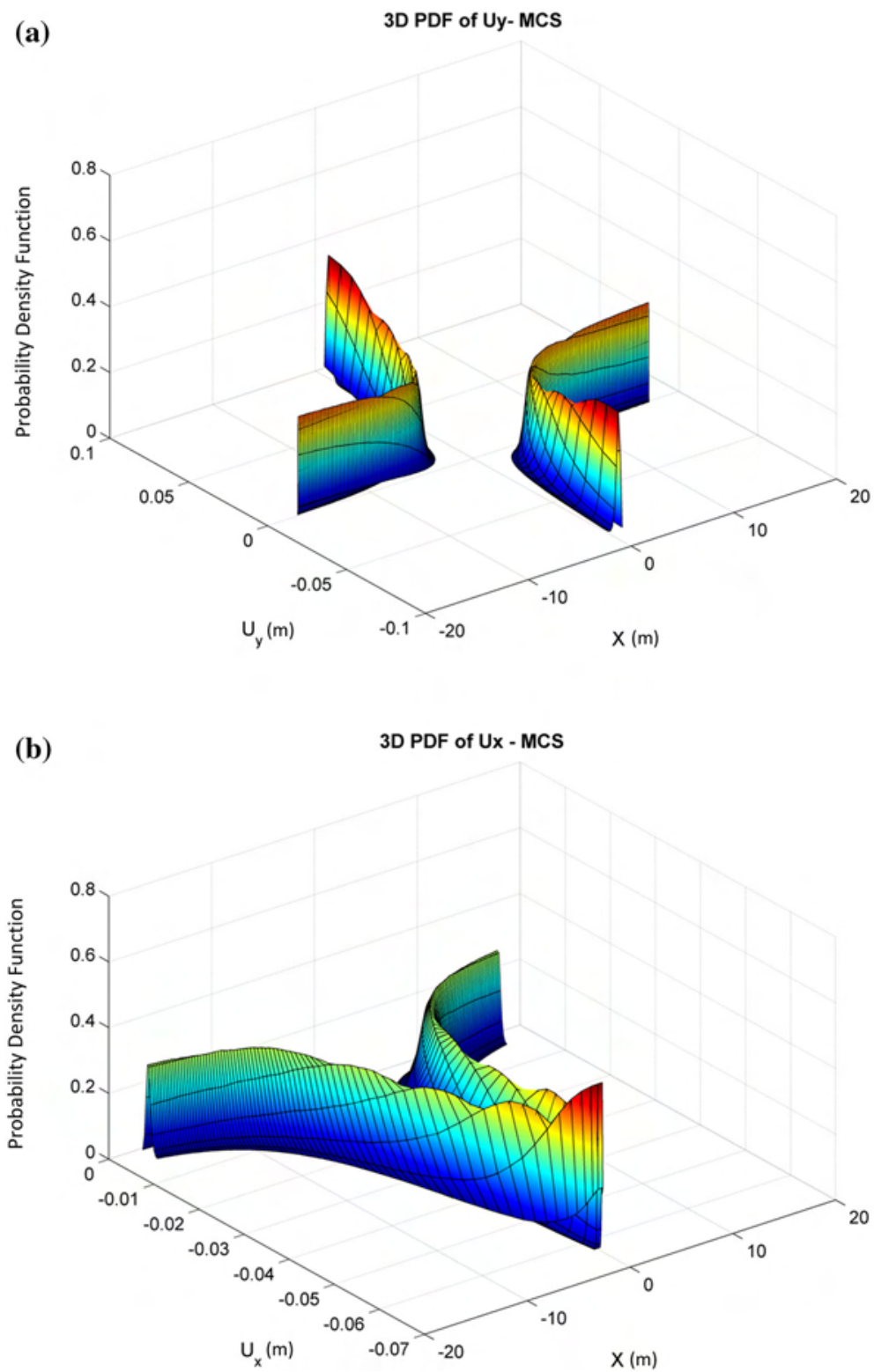

Figure 10. Probability density functions of ground surface response using the MCS-based AS for (a) vertical and (b) horizontal displacements. 
MCS-based SNT-FEM are provided in figure 3 by a flowchart.

\subsection{MCS-based AS}

The main difference between the MCS-based AS and the MCS-based SNT-FEM is that the analysis part is devoted to existing closed-form solutions in the MCS-based AS procedure. Thus, a fast computation is accomplished, and the solutions are obtained by exact formulations. Nevertheless, this method can only be used for the geometry and properties having analytical solutions.
In other words, the method is not versatile for every complex dislocation problem. In figure 4 , a flowchart is given to illustrate the procedure of the MCS-based AS.

\section{Illustrative examples}

\subsection{A vertical outcropped fault}

For this illustrative example, the fault length is randomly generated at each simulation by the following relationship

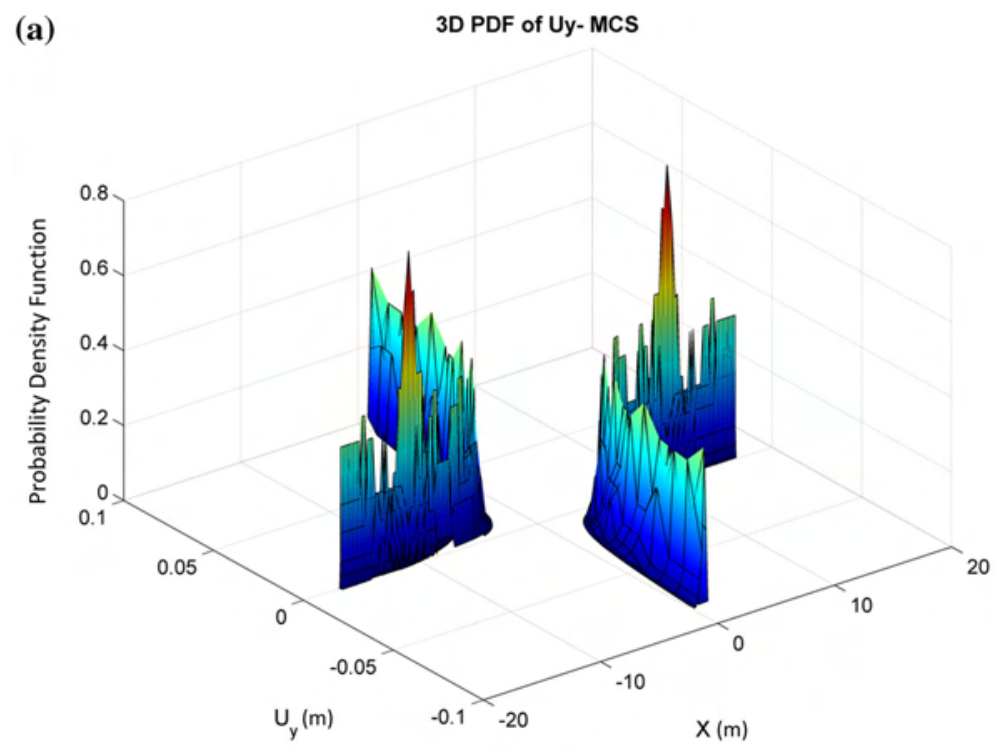

(b) 3D PDF of Ux - MCS

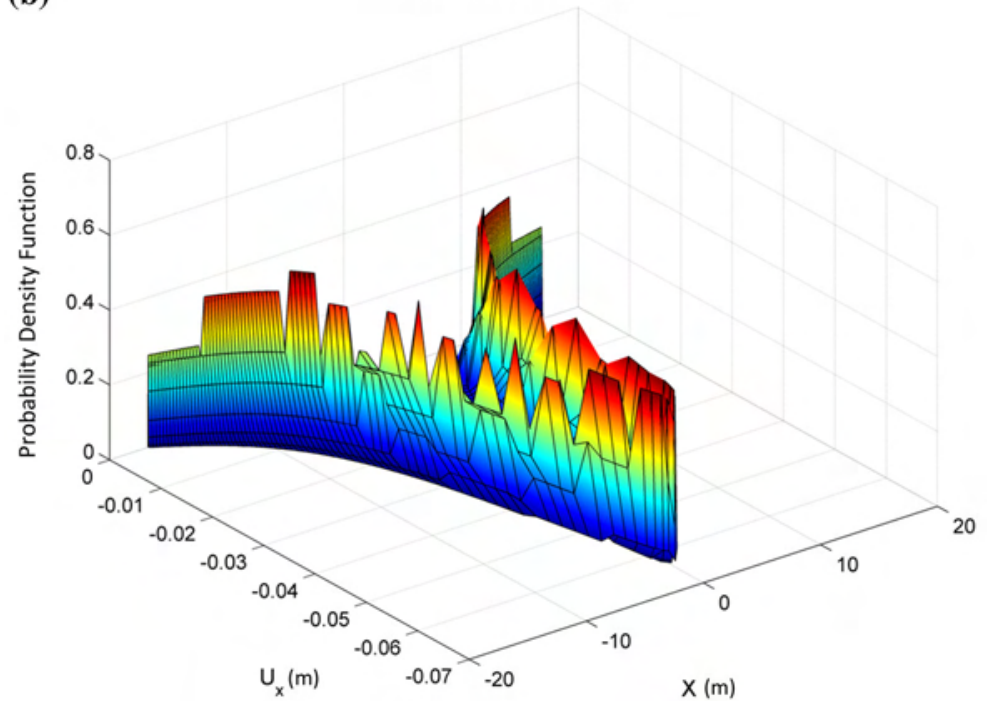

Figure 11. Probability density functions of ground surface response using the MCS-based SNT-FEM for (a) vertical and (b) horizontal displacements. 


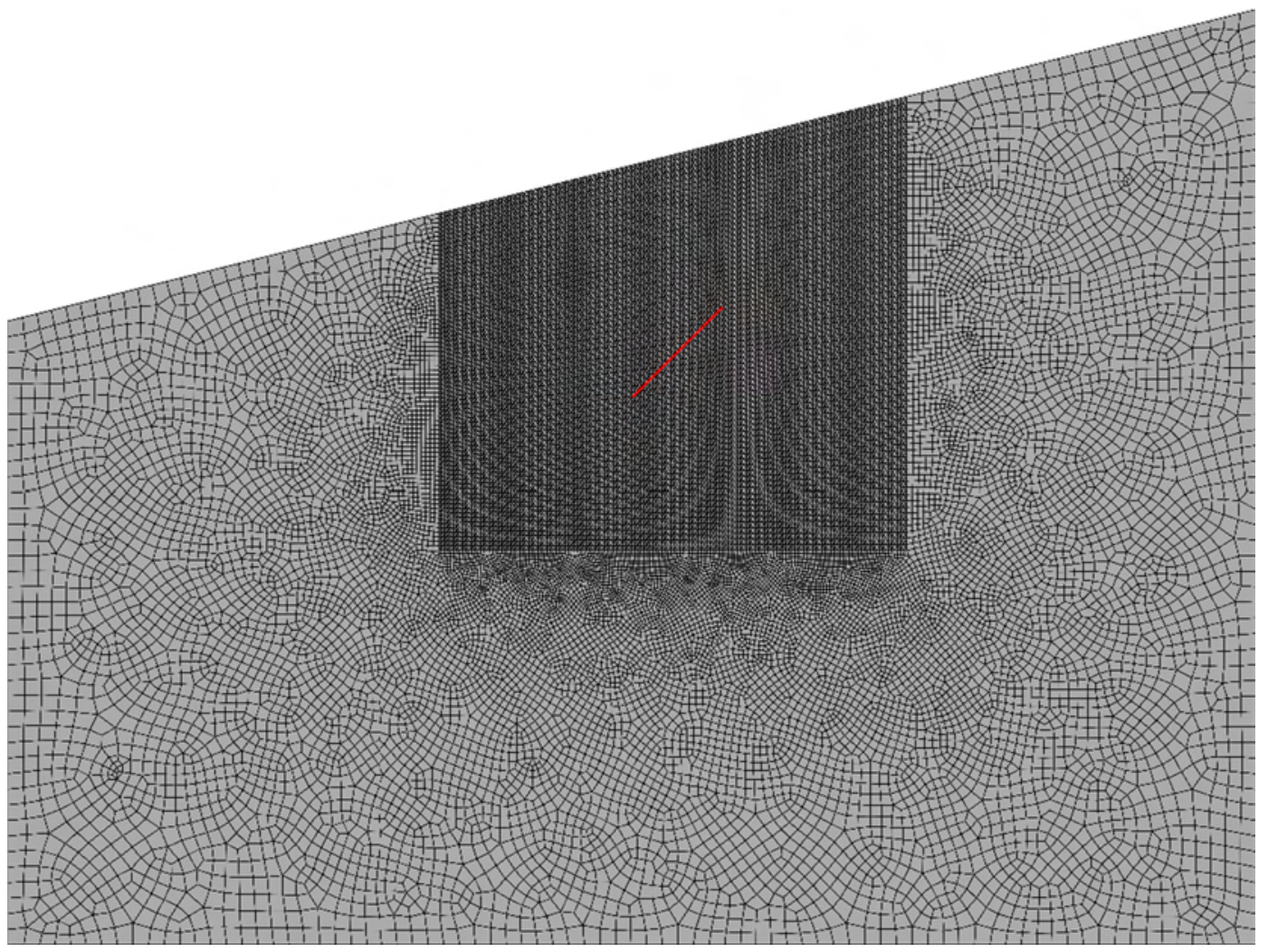

Figure 12. The inclined embedded fault shown by red solid line in a steep layered domain. The finite element mesh of the domain contains triangular and quadrilateral elements.

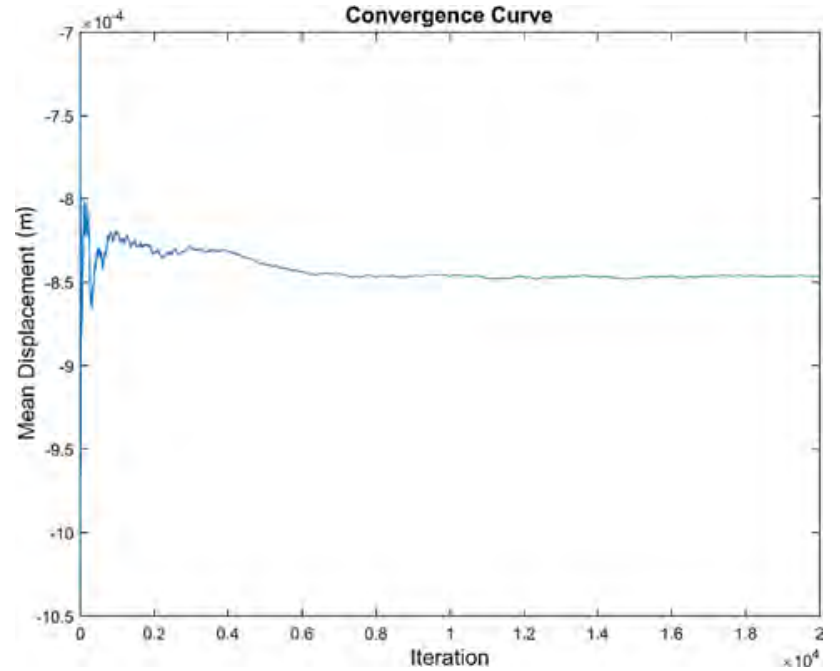

Figure 13. Convergence curve of the MCS-based SNT-FEM for mean value of an arbitrary DOF.

$$
L=L_{m}+\operatorname{rand} \times \sigma_{L}
$$

where rand is assumed to be a normally distributed random variable, $L_{m}$ and $\sigma_{L}$ are mean length and its standard deviation, respectively. Response statistics of a variable such as $u$ is obtained by

$$
u_{m}=\frac{1}{n} \sum_{i=1}^{n} u_{i}
$$

and

$$
\sigma_{m}=\sqrt{\frac{1}{n-1} \sum_{i=1}^{n}\left(u_{i}-u_{m}\right)^{2}}
$$

where $u_{i}$ is a response at the $i$ th simulation. Furthermore, $u_{m}, \sigma_{m}$, and $n$ represent the mean value, the standard deviation value, and the number of samples (or simulations), respectively. Here, the length of the fault has normal distribution, for which the mean and standard deviation values of the fault length are chosen as 3 and $0.4 \mathrm{~m}$, respectively. In addition, the total slip is $0.2 \mathrm{~m}$, and the Poisson's ratio is taken as 0.25 . The vertical fault of this example is located at the middle of the domain where its fixed top end reaches to ground surface, as shown in figure 5 . In the case of numerical analysis, a FE mesh of $150 \times 82$ quadrilateral 4-node elements with plane strain condition is considered for the domain with dimension of $34 \times 19 \mathrm{~m}$. Both MCS analyses are accomplished with 10000 simulations. In addition, the elasticity modulus of the domain is selected as 1.24E4 MPa. 
The response mean and standard deviation values of ground surface are drawn in figures 6 and 7 , which imply that the response curves show remarkable agreements. Furthermore, the mean and standard deviation of vertical and horizontal displacement fields obtained by the MCS-based SNT-FEM are illustrated in figures 8 and 9. In these figures, the response values are represented as contours in the deformed form of the domain. In addition, the probability density functions (PDFs) of the responses at ground surface are indicated by $3 \mathrm{D}$ surfaces in figure 10, which resulted from the MCS-based AS. It should be noted that 3D representations are collection of PDFs of displacement responses at every point of ground surface according to the grid interval size. In the case of MCS-based SNT-FEM, figure 11 provides the corresponding PDF functions. Obviously, the numerical approach may not capture exact responses as suitable as analytical approach. In other words, there are some reasonable minor errors in the SNT-FEM approach with respect to
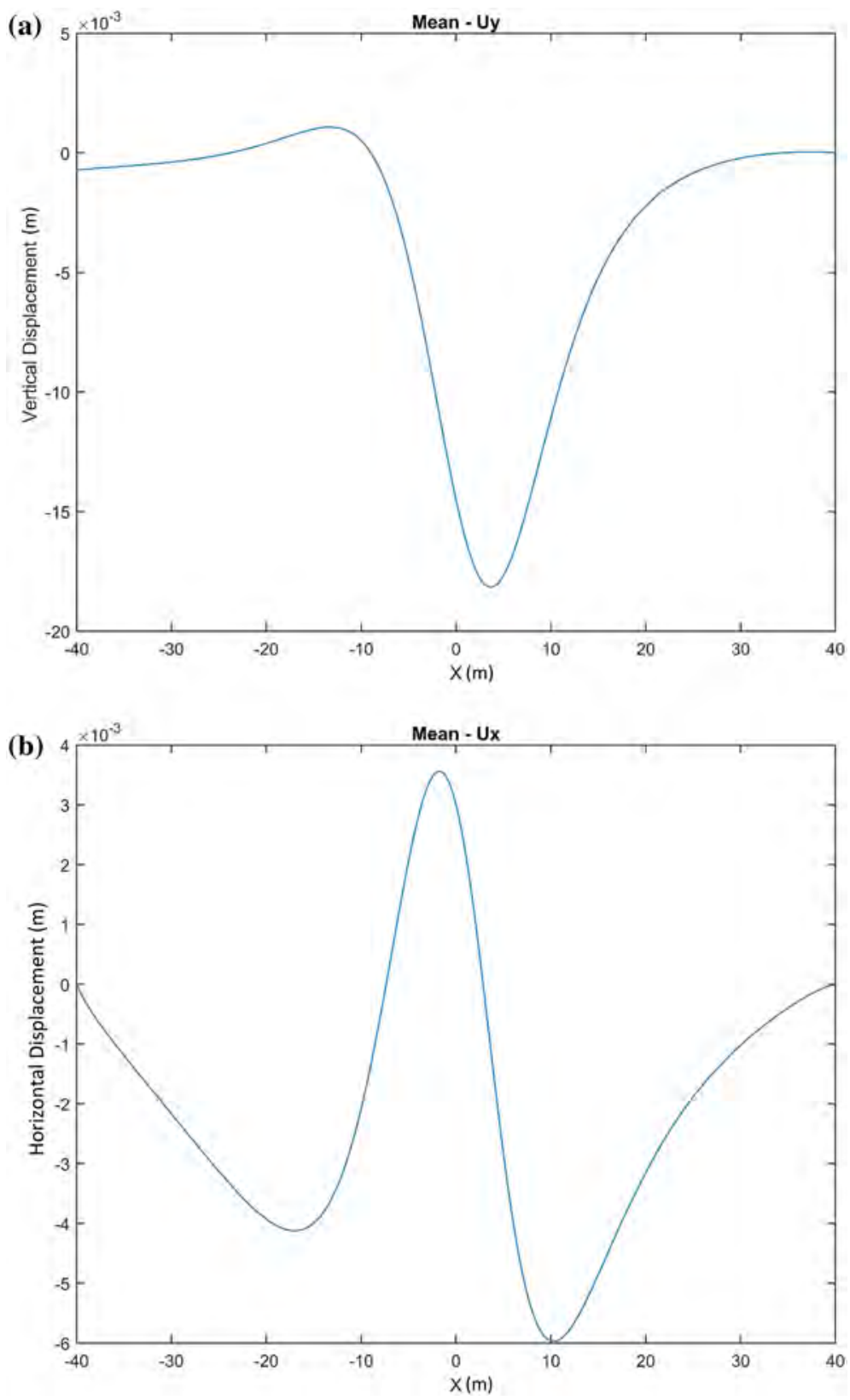

Figure 14. Response mean values of ground surface: (a) vertical displacement, and (b) horizontal displacement. 

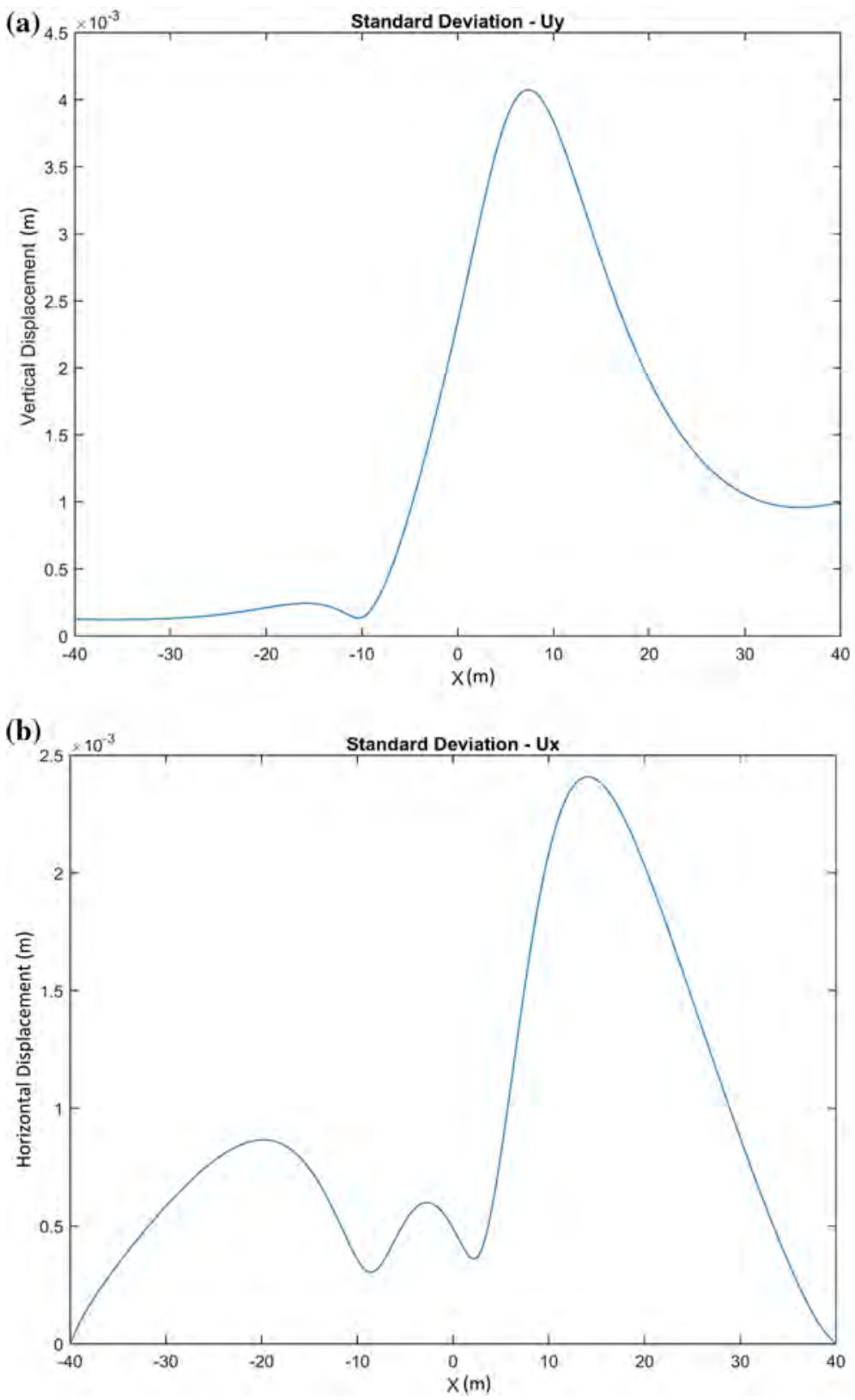

Figure 15. Response standard deviation values of ground surface: (a) vertical displacement and (b) horizontal displacement.

the AS one, and these errors clearly magnifies for the PDFs and higher order statistical moments (e.g., standard deviations). Another source of this error is the rounding error existing in the MCSbased SNT-FEM so that the generated fault length due to the realization should be rounded to the nearest element node at each simulation. However, this phenomenon may be resolved by selecting a finer mesh or performing adaptive re-meshing, which is not in the scope of the present work.

\subsection{An inclined embedded fault in a layered domain}

This example deals with a two layered domain having an inclined embedded fault (figure 12) where its length and orientation are randomly being generated at each simulation. Four corners of the domain have coordinates $(-40,-20),(40$, $20),(40,40)$ and $(-40,20)$ meters in counterclockwise direction. The uniform thickness of the bottom layer (the second one) is $38 \mathrm{~m}$ consisting of 
(a)

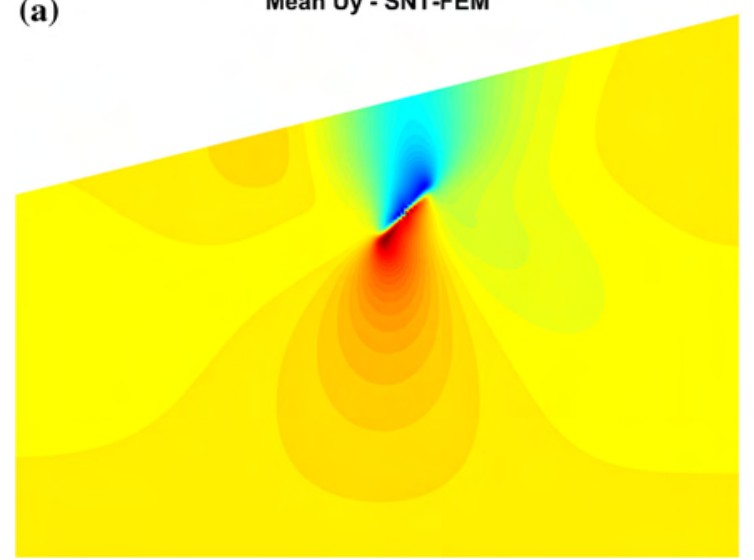

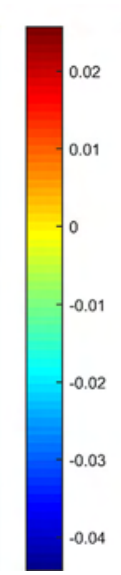

(b)

Mean Ux - SNT-FEM

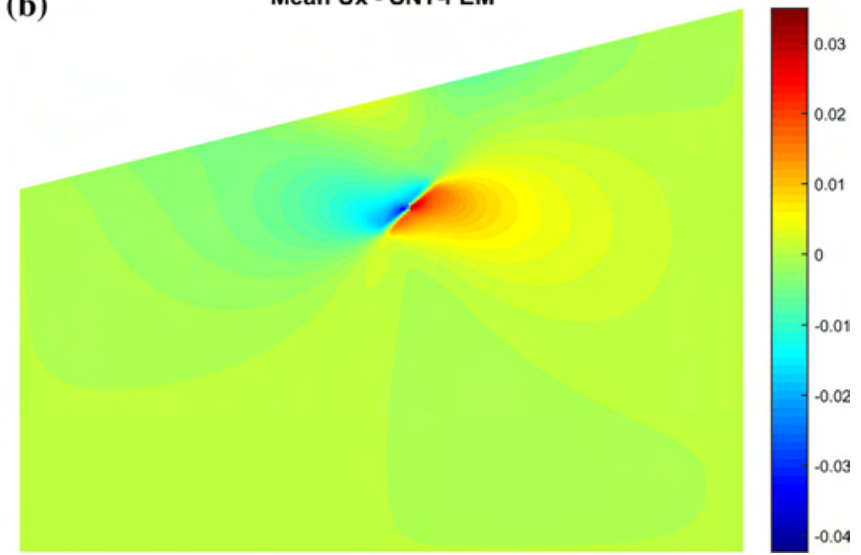

Figure 16. Displacement field contours of mean values in the domain: (a) vertical and (b) horizontal displacements (in $\mathrm{m}$ ).

(a)

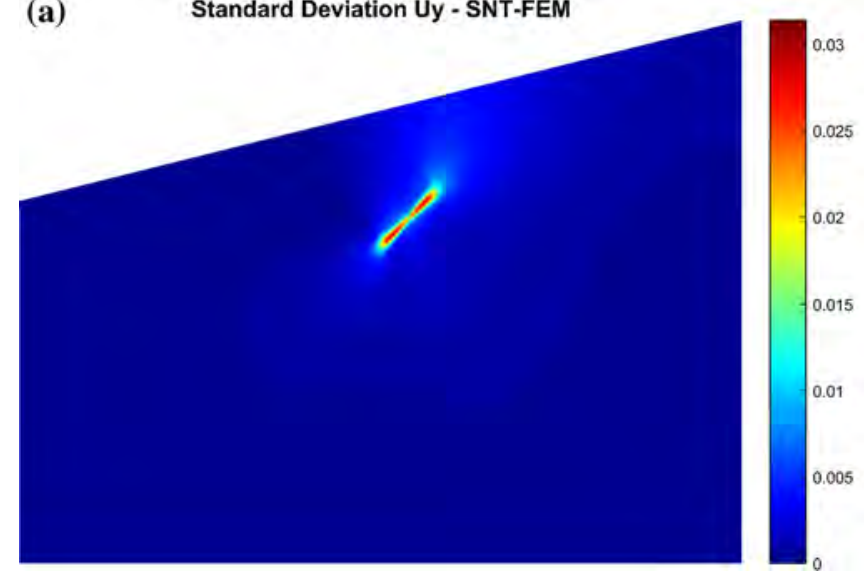

(b)

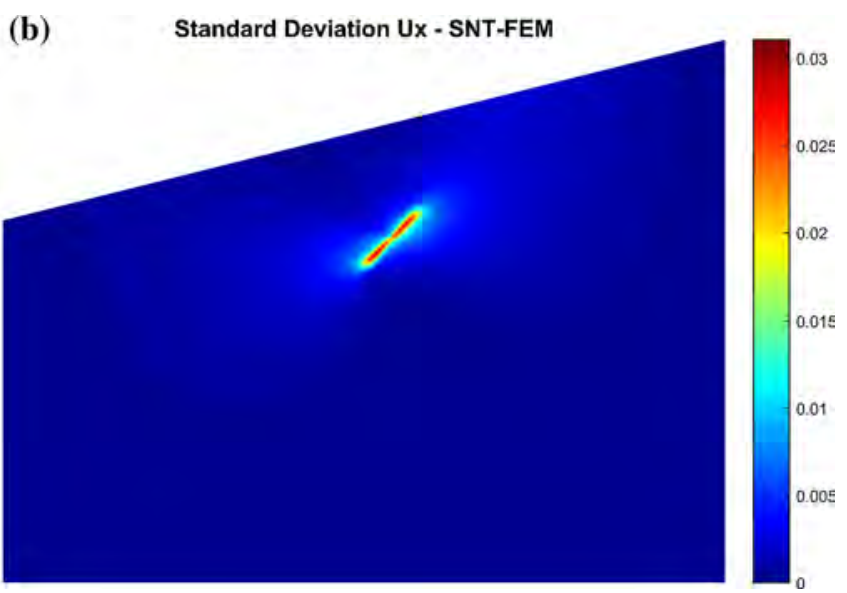

Figure 17. Displacement field contours of standard deviation values in the domain: (a) vertical and (b) horizontal displacements (in $\mathrm{m}$ ).

elastic modulus of $2 \mathrm{E} 10 \mathrm{kN} / \mathrm{m}^{2}$ and Poisson's ratio of 0.3 . Coordinates of the fault center is equal to $(3 \mathrm{~m}, 18 \mathrm{~m})$ assumed to be fixed in all iterations, while the interface line also passes through this point. The remaining part of the domain is the first layer with elastic modulus of $1.24 \mathrm{E} 10 \mathrm{kN} / \mathrm{m}^{2}$ and Poisson's ratio of 0.25 . Slip magnitude is assumed to be $0.2 \mathrm{~m}$ under plane strain condition. Orientation of the fault is obtained as given by

$$
\theta=\theta_{m}+\operatorname{rand} \times \sigma_{\theta}
$$

where $\theta_{m}$ and $\sigma_{\theta}$ imply mean fault angle with respect to horizontal line and its standard deviation, respectively. Length and orientation angle of the fault are supposed to be independent Gaussian random variables. Mean and standard deviation values of the fault's length are equal to 8 and $0.8 \mathrm{~m}$, respectively. Furthermore, mean and standard deviation values of the fault's orientation angle are taken as $\pi / 4$ and $\pi / 36$, respectively. This example is a more realistic case which does not have any closed-form solution, and therefore it is solved by MCS-based SNT-FEM, solely. Furthermore, this example is another demonstration to indicate capability and suitability of MCS-based SNT-FEM for complex domains. In order to overcome this kind of large domain with suitable accuracy and convergence, very small triangular elements are employed for the part including randomly generated faults, while quadrilateral elements are utilized for the far field of the domain. Therefore, the distorted mesh includes 35,196 finite elements. In each simulation as a vital task, the fault line, split nodes and 
contributing elements (split elements) should be found, and then the force vector should be remade for calculations followed by the simulation at each iteration.

After performing 20,000 simulations, convergence curve for mean value of an arbitrary DOF is plotted in figure 13, showing assurance for the MCS convergence. Surface displacement responses of the domain are depicted in figures 14 and 15 . Mean and standard deviation of response fields of the whole domain are illustrated in figures 16 and 17 . These results illustrate that whenever the fault length and orientation are uncertain due to lack of knowledge and measurement problems, one may estimate the response through reliability approaches like MCS-based SNT-FEM, as uncertainty-induced effects are inevitable, particularly in seismology.

PDFs of the responses at surface nodes are depicted as 3D plots in figure 18. As another interpretation, each $3 \mathrm{D}$ plot is a set of $2 \mathrm{D}$ probability density functions for every surface node response with different skewness and kurtosis which are viewed together in the $3 \mathrm{D}$ plot. Clearly, these are
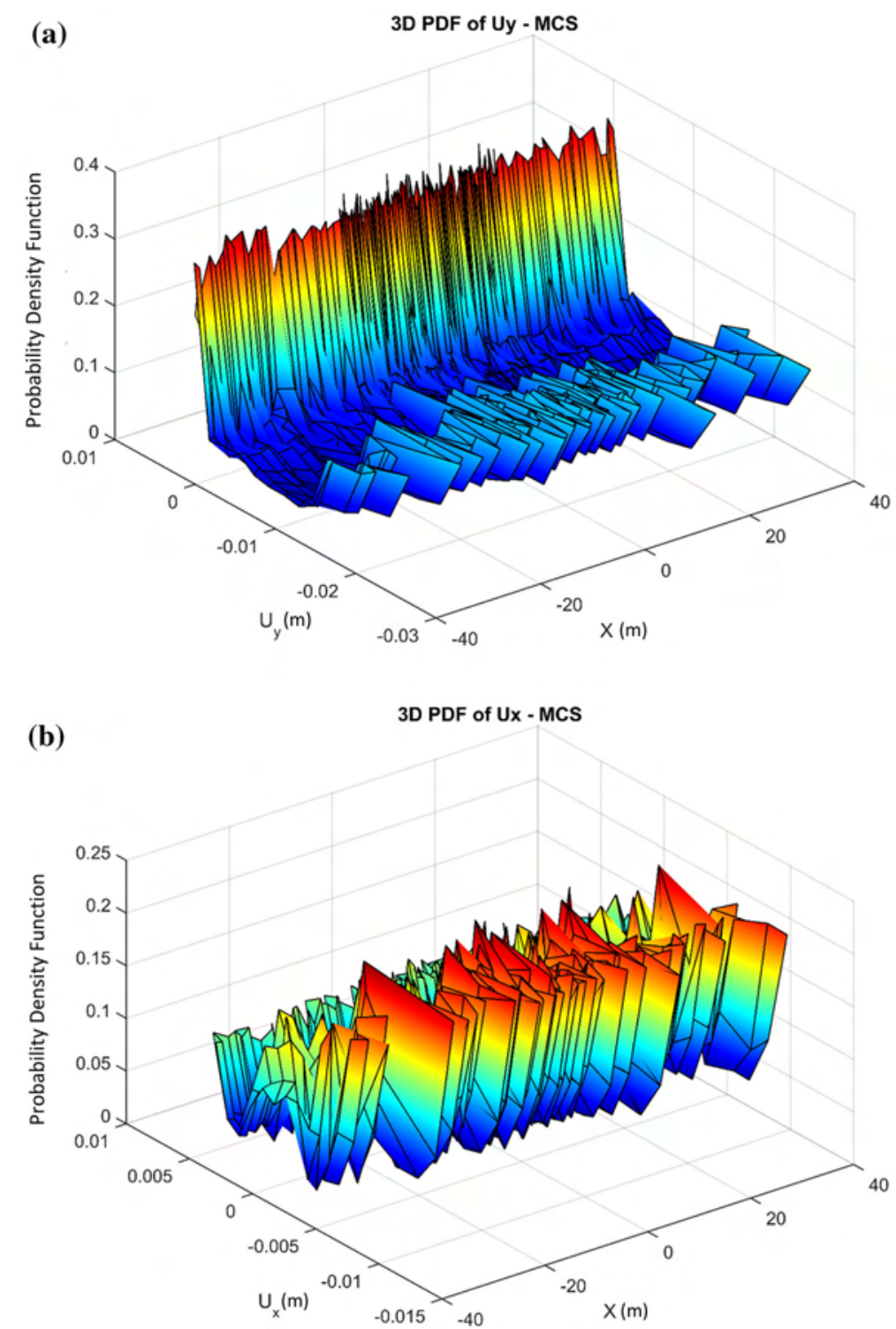

Figure 18. Probability density functions of ground surface response using the MCS-based SNT-FEM for (a) vertical and (b) horizontal displacements. 
joint PDFs, and as there are two independent random variables, they can be separately written as multiples of single variable.

Consequently, the MCS-based SNT-FEM is a robust alternative to the MCS-based AS for cases with no analytical solution. Although it had been shown that the SNT-FEM is a useful method for fault dislocations, particularly for complicated geometries (van Zwieten et al. 2013), the obtained results demonstrate that the MCS-based SNTFEM can also maintain this ability in random geometry. As a result, the SNT may be generalized to stochastic modeling of earthquake fault dislocation problems.

\subsection{A model of San Fernando fault}

The final example is a more practical application of the proposed method. For briefness, only important figures are indicated for this example. Since there is no practical benchmark example for verification
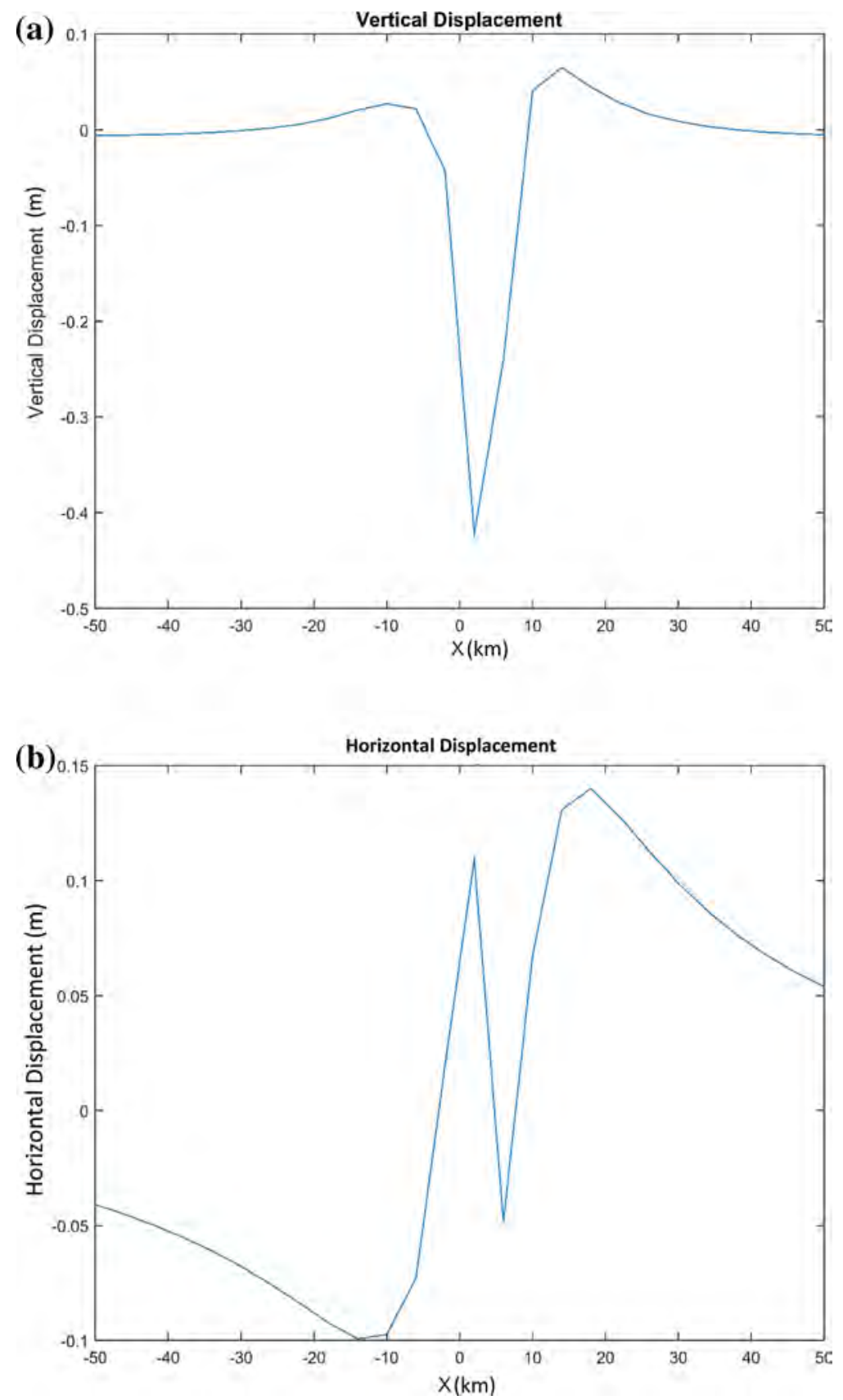

Figure 19. Deterministic solution of the benchmark example using the SNT-FEM for (a) vertical surface displacement and (b) horizontal surface displacement. 
of this kind of stochastic modeling, three steps are followed in this section.

First, deterministic solution of a benchmark example (Megna et al. 2005) is solved here and drawn in figure 19 which confirms the results of Megna et al. (2005), successfully.
Second, deterministic solution of San Fernando fault (see figure 20) is obtained. Table 1 shows martial properties of the model, which are obtained from Toki and Sawada (1988) and Jungels and Frazier (1973). Figure 21 illustrates the results of deterministic solution of San Fernando fault, for

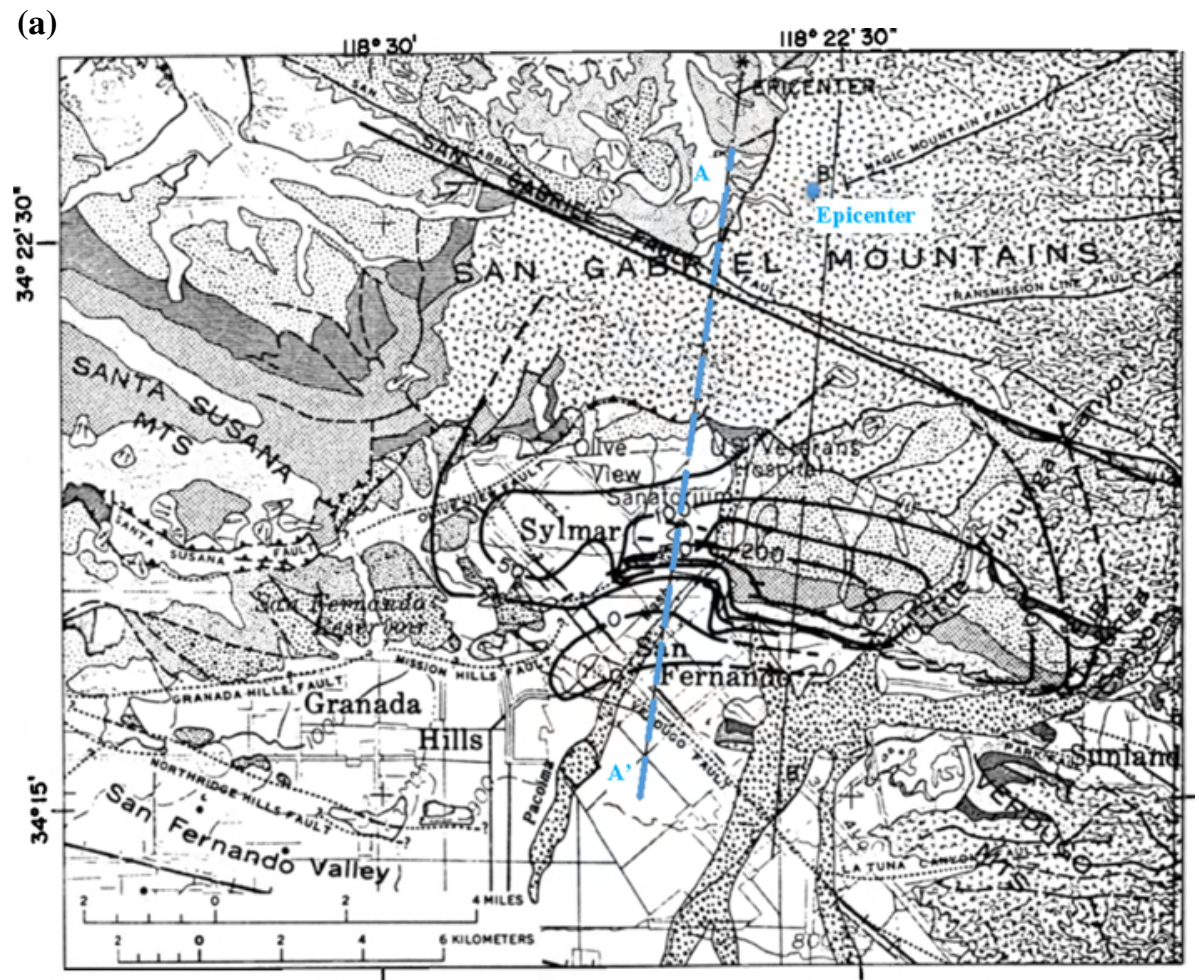

(b)

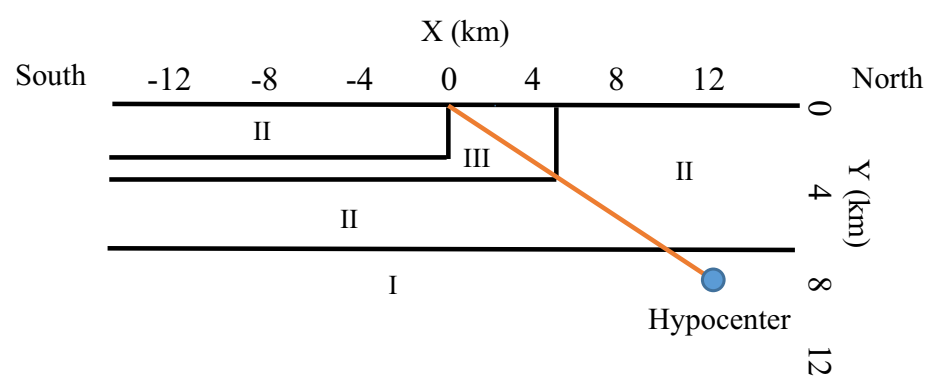

Figure 20. (a) Geologic map of San Fernando earthquake (Jungels and Frazier 1973), and (b) configuration of AA' section (indicated by blue long dash line) containing a San Fernando earthquake fault (indicated by orange line).

Table 1. Material properties of the crustal layers for San Fernando fault.

\begin{tabular}{lccc}
\hline Layer & $\begin{array}{c}\text { Mass density } \\
\left(\mathrm{kg} / \mathrm{m}^{3}\right)\end{array}$ & $\begin{array}{c}\text { Shear wave } \\
\text { velocity }(\mathrm{m} / \mathrm{s})\end{array}$ & Poisson's ratio \\
\hline I & 3000 & 3400 & 0.27 \\
II & 2700 & 3400 & 0.24 \\
III & 2500 & 2500 & 0.22 \\
\hline
\end{tabular}



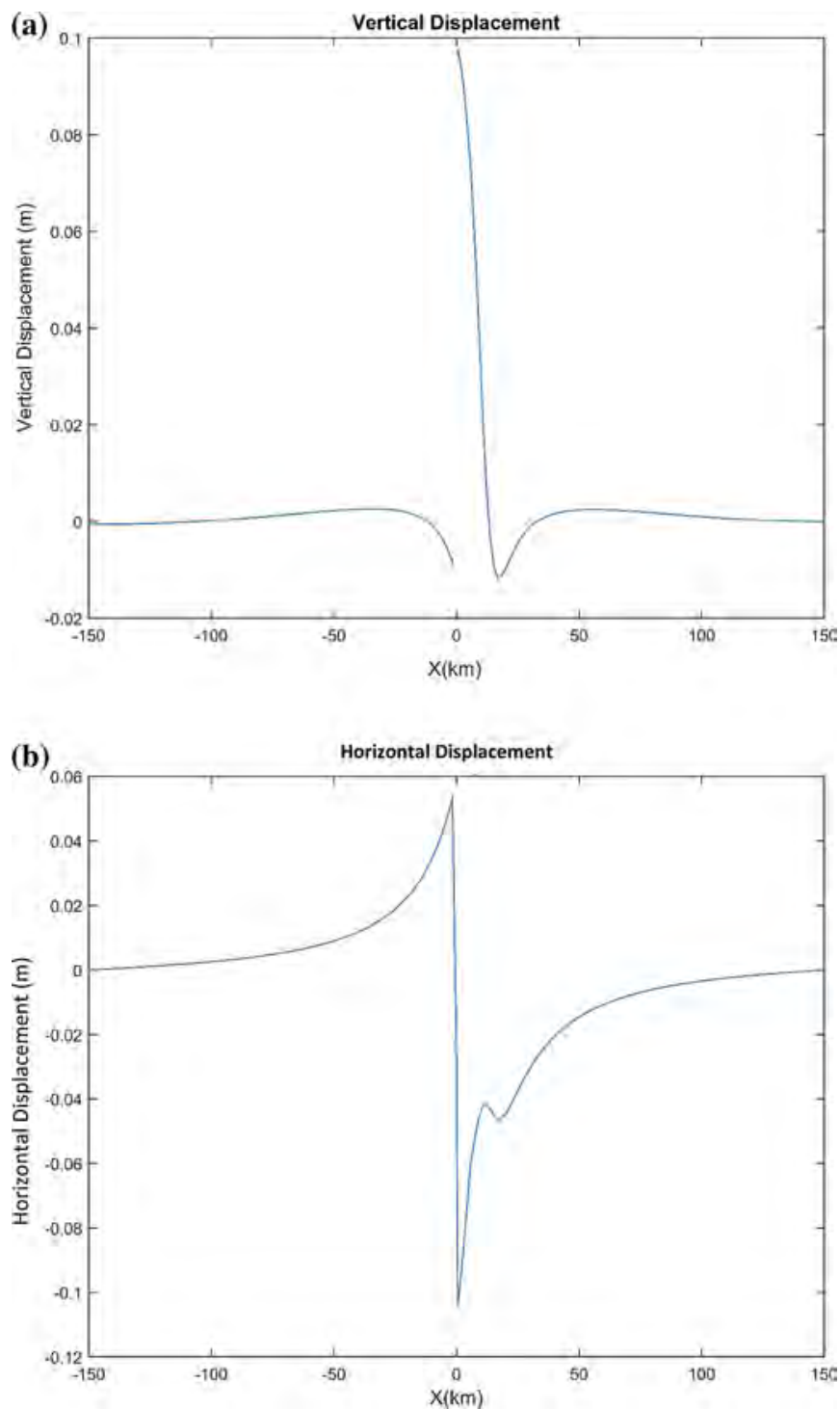

Figure 21. Deterministic solution of San Fernando fault under $0.2 \mathrm{~m}$ slip using the SNT-FEM for (a) vertical surface displacement, and (b) horizontal surface displacement.

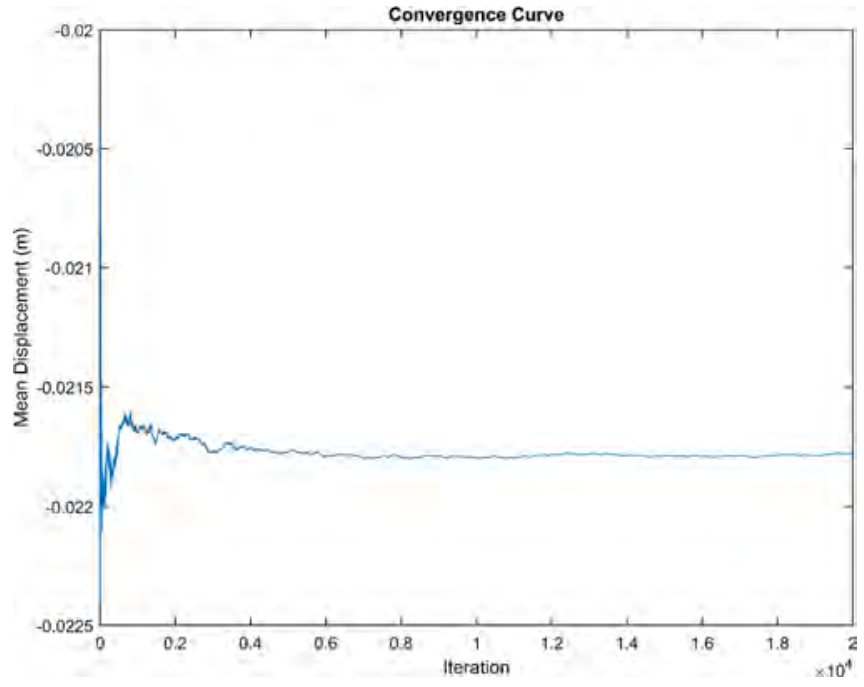

Figure 22. Convergence curve of the MCS-based SNT-FEM for mean value of an arbitrary DOF in San Fernando fault numerical analysis. 

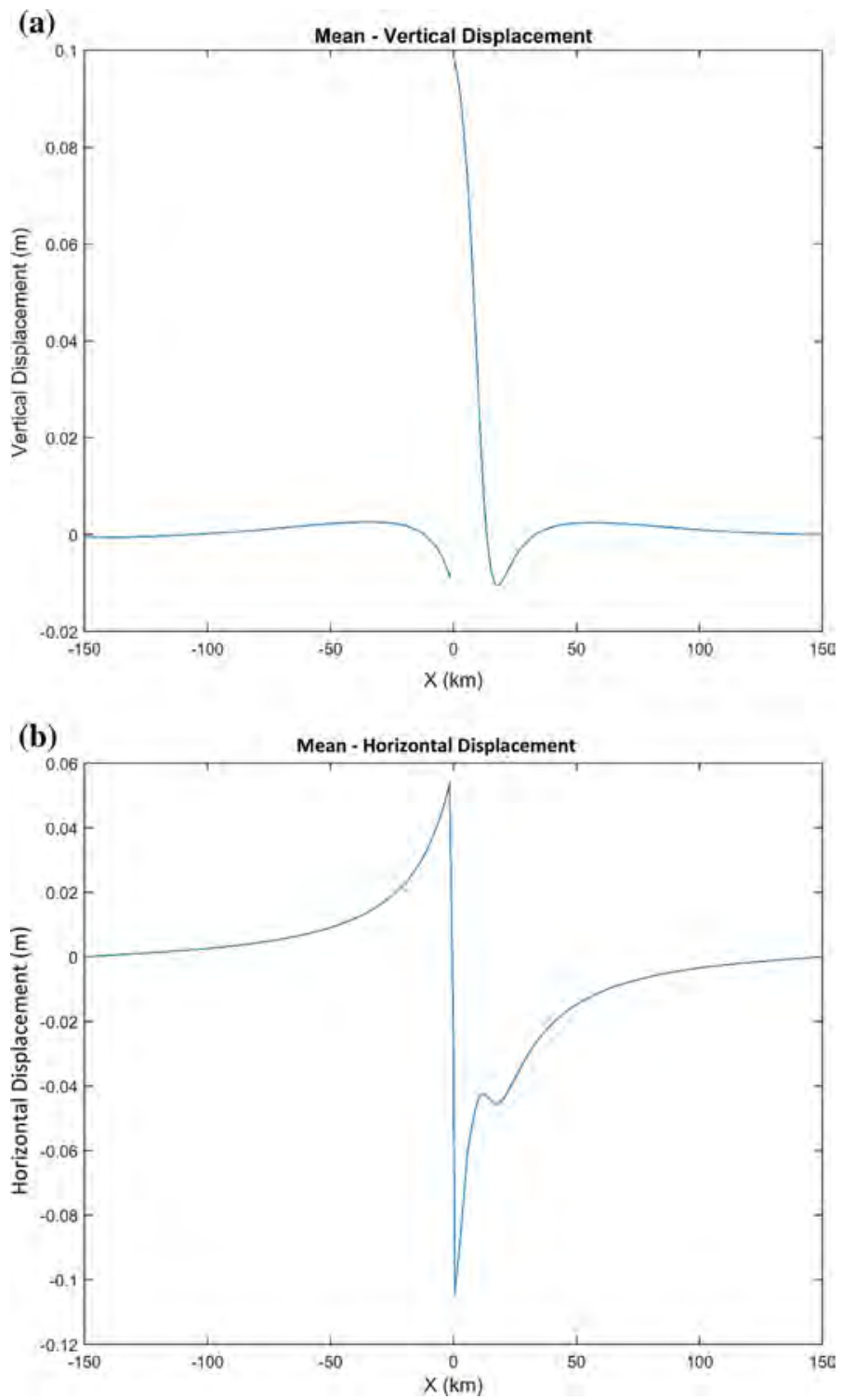

Figure 23. Response mean values of ground surface due to San Fernando fault slip: (a) vertical displacement and (b) horizontal displacement.

which the SNT-FEM uses a uniform slip of $0.2 \mathrm{~m}$ similar to the previous examples for sake of inherent features in the formulations. In this numerical model, 22,398 plane strain triangular elements are utilized for the section depicted in figure 20(b).

Third, mean values of the MCS-based SNTFEM are often nearly identical to the deterministic solution, so this confirms the results of the MCS. Length of the fault at its internal end is a random variable with $10 \%$ standard deviation. In other words, uncertain place of hypocenter at fault line direction is considered. The same finite element mesh and domain $(150 \times 80 \mathrm{~km})$ of deterministic analysis is used for the MCS-based SNT-FEM. In order to show a complete response decay and eliminate boundary effects, large domain dimensions are considered here. Mean response of an arbitrary node is used for the convergence curve of MCS-based SNT-FEM as drawn in figure 22. This example and the previous one show that one 

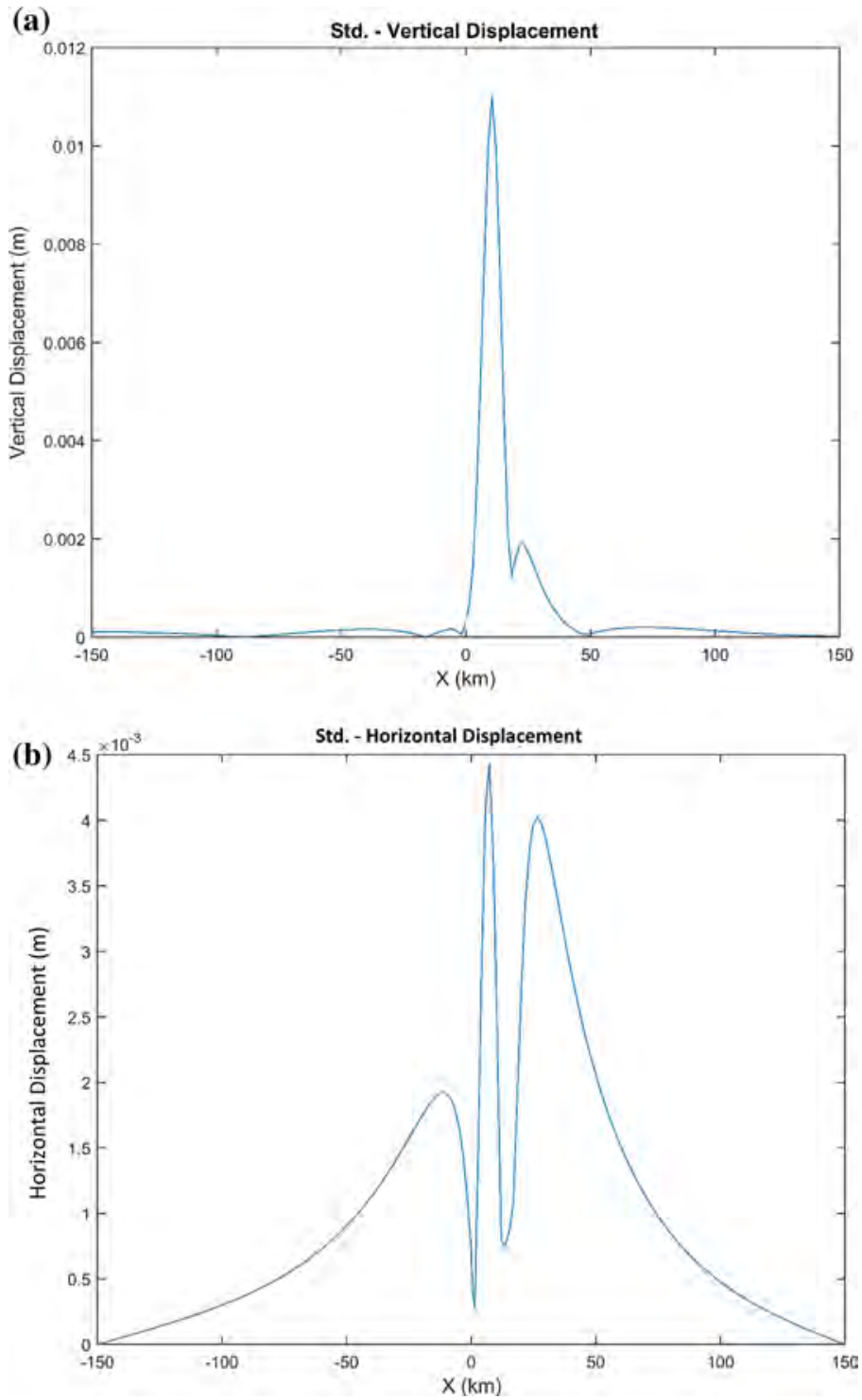

Figure 24. Response standard deviation values of ground surface due to San Fernando fault slip: (a) vertical displacement and (b) horizontal displacement.

may obtain suitable convergence by performing 10,000 simulations rather than 20,000. Mean values of surface response are illustrated in figure 23 , which may be validated by deterministic solution of figure 21. Standard deviations of surface response are plotted in figure 24. Also, figures 25 and 26 indicate mean values and standard deviations of field displacements. In addition, PDFs of responses are represented in figure 27. Conciseness of a developing research does not allow to report all aspects of a study, however this is worth mentioning that it is more practical to find PDF of each location (desired points) of the domain separately, and use finer meshes around the desired point(s) in order to have a better and accurate PDF for further applications in accordance with the reasons explained at the last parts of the first example. 

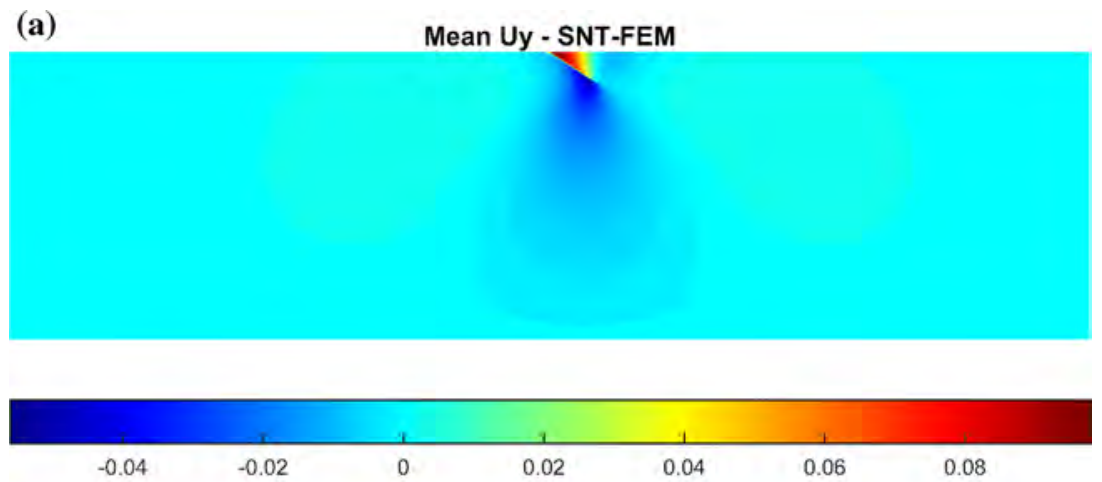

(b)

Mean UX - SNT-FEM

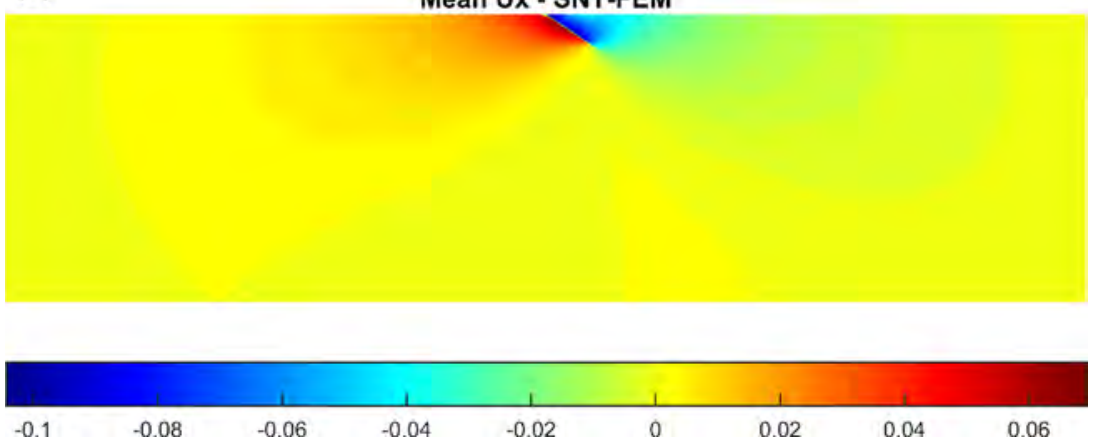

Figure 25. Displacement field contours of mean values in the San Fernando domain: (a) vertical displacement and (b) horizontal displacement (in $\mathrm{m}$ ).
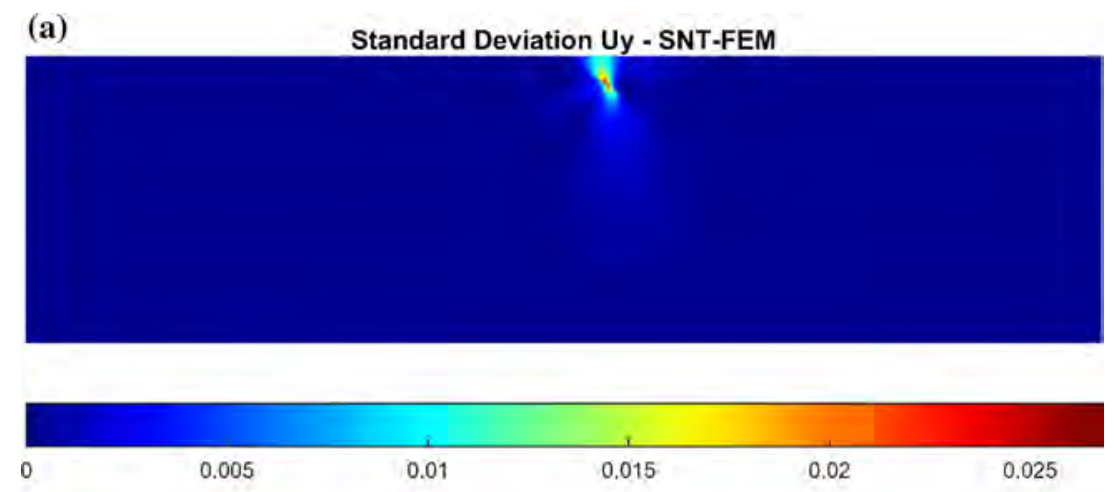

(b)

Standard Deviation UX - SNT-FEM
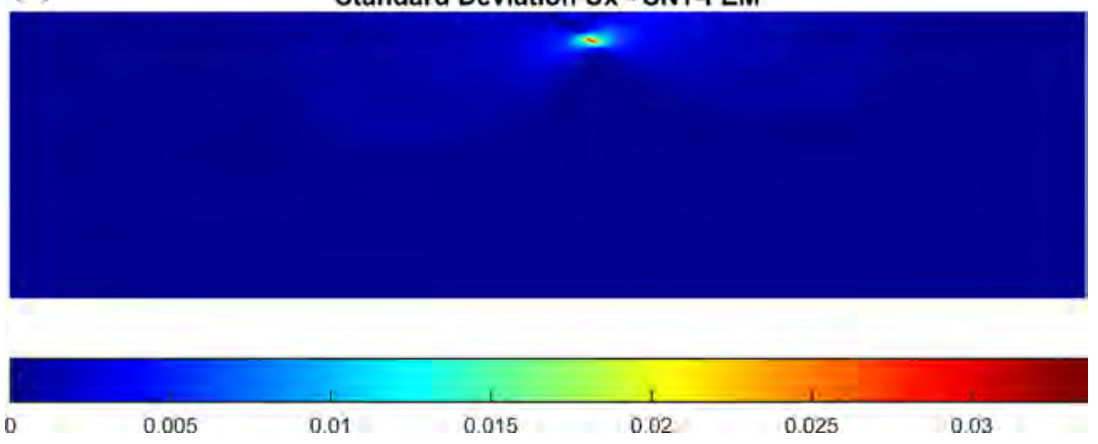

Figure 26. Displacement field contours of standard deviation values in the San Fernando domain: (a) vertical displacement and (b) horizontal displacement (in $\mathrm{m}$ ). 

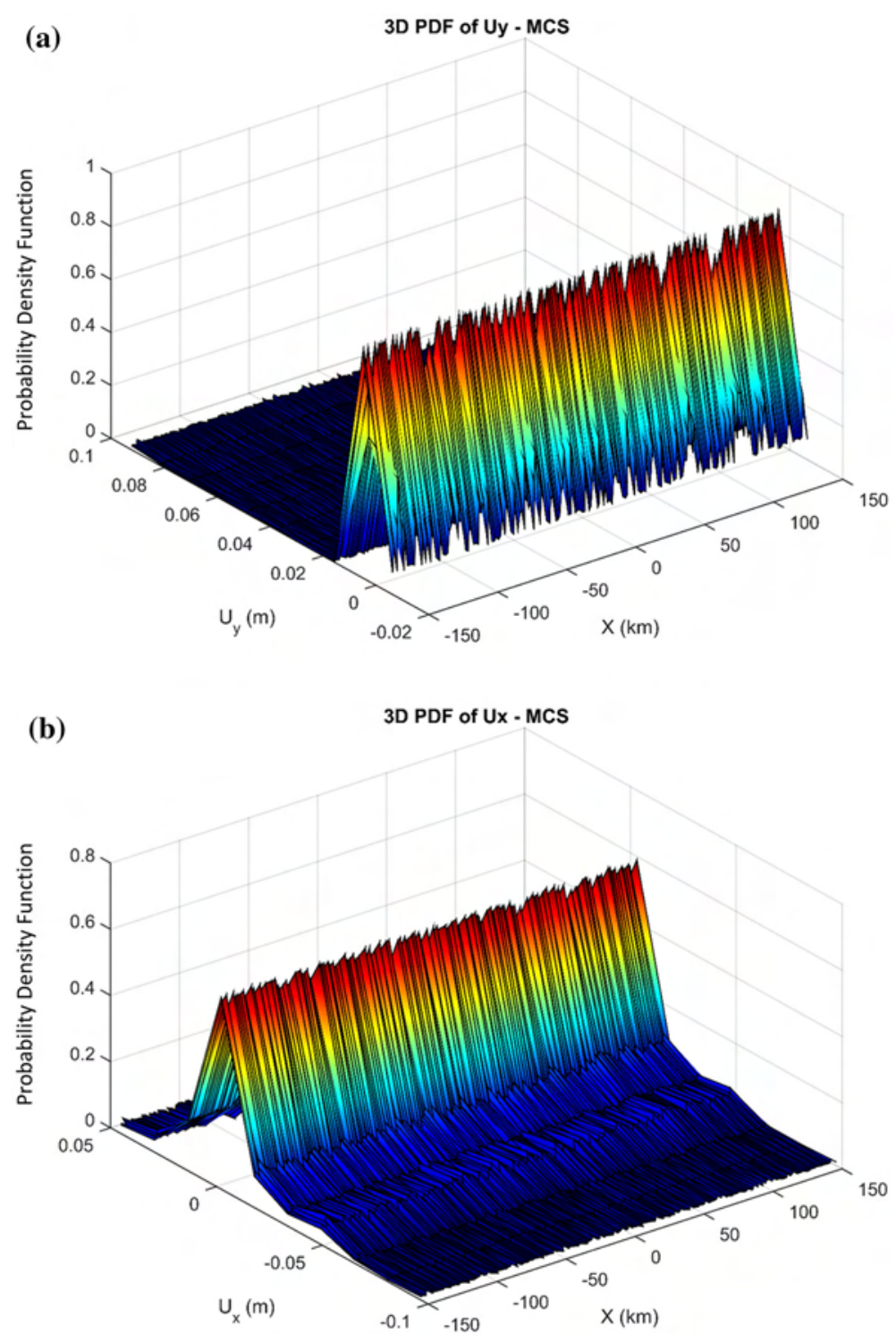

Figure 27. Probability density functions of ground surface response using the MCS-based SNT-FEM for (a) vertical, and (b) horizontal displacements of San Fernando fault.

\section{Conclusions}

Earthquake fault dislocation computation is a vital task in computational earthquake engineering, as potential earthquakes' quantities may be estimated from fault dislocation. Furthermore, geological properties of an active fault cannot be measured precisely without uncertainties. This study presents a MCS-based random simulations of fault dislocation to consider geometrical uncertainty. Two approaches are utilized, including numerical method which uses the FEM incorporating the SNT, and analytical method, to impose the effects of discontinuities. Length and orientation angle of the fault are taken as random variables in the models. Mean values, standard deviation values, and probability density functions of ground surface responses caused by the dislocation are computed. The capability, accuracy, and viability of the proposed methods (i.e., the MCS-based AS and the MCS-based SNT-FEM) are concluded from the results for random simulation of fault dislocation. The development of the present method for its application in more practical fault geometries is currently being followed by the authors, and the obtained results may be published soon. 


\section{Acknowledgements}

The authors wish to acknowledge and express their special gratitude to two anonymous reviewers, for their constructive advices that improved the manuscript.

\section{References}

Andrews D J 1999 Test of two methods for faulting in finite-difference calculations; Bull. Seismol. Soc. Am. 89 931-937.

Dalguer L A and Day S M 2006 Comparison of fault representation methods in finite difference simulations of dynamic rupture; Bull. Seismol. Soc. Am. 96 1764-1778.

Dalguer L A and Day S M 2007 Staggered-grid split-node method for spontaneous rupture simulation; J. Geophys. Res. - Solid Earth 112 B02302.

Hainzl S, Zöller G, Brietzke G B and Hinzen K G 2013 Comparison of deterministic and stochastic earthquake simulators for fault interactions in the Lower Rhine Embayment Germany; Geophys. J. Int. 195 684-694.

Hori M, Ichimura T and Nakagawa H 2003 Analysis methods of stochastic model: Application to strong motion and fault problems; Struct. Eng. Earthq. Eng. 20 105s-118s.

Hori M 2011 Introduction to Computational Earthquake Engineering; World Scientific Publishers, Singapore.

Kagan Y Y 1982 Stochastic model of earthquake fault geometry; Geophys. J. Roy. Astron. Soc. 71 659-691.

Jungels P H and Frazier G A 1973 Finite element analysis of the residual displacements for an earthquake rupture: Source parameters for the San Fernando Earthquake; J. Geophys. Res. 78 5062-5083.

Manglik A, Thiagarajan S, Mikhailova A V and Rebetsky Yu 2008 Finite element modelling of elastic intraplate stresses due to heterogeneities in crustal density and mechanical properties for the Jabalpur earthquake region, central India; J. Earth Syst. Sci. 117 103-111.
Megna A, Barba S and Santini S 2005 Normal-fault stress and displacement through finite-element analysis; Ann. Geophys. 48 1009-1016.

Melosh H J and Raefsky A 1981 A simple and efficient method for introducing faults into finite element computations; Bull. Seismol. Soc. Am. 71 1391-1400.

Melosh H J and Raefsky A 1983 Anelastic response of the Earth to a dip-slip earthquake; J. Geophys. Res. - Solid Earth 88 515-526.

Melosh H J and Williams C A 1989 Mechanics of graben formation in crustal rocks: A finite element analysis; $J$. Geophys. Res. - Solid Earth 94 13,961-13,973.

Okada Y 1992 Internal deformation due to shear and tensile faults in a half-space; Bull. Seismol. Soc. Am. 82 1018-1040.

Rani S and Singh S J 1992 Static deformation of a uniform half-space due to a long dip-slip fault; Geophys. J. Int. 109 469-476.

Rani S and Bala N 2013 Deformation of a two-phase medium due to a long buried strike-slip fault; Nat. Sci. 51078.

Toki K and Sawada S 1988 Simulation of the fault rupture process and near field ground motion by the threedimensional finite element method; In: Proc. 9th World Conf. Earthquake Engineering, August 2-9, 1988, TokyoKyoto, Japan.

Toki K, Sawada S and Okashige Y 1987 Simulation of fault rupture process by the stochastic finite element method; Probabilist. Eng. Mech. 2 129-137.

van Zwieten G J, Hanssen R F and Gutiérrez M A 2013 Overview of a range of solution methods for elastic dislocation problems in geophysics; J. Geophys. Res. - Solid Earth 118 1721-1732.

van Zwieten G J, van Brummelen E H, van der Zee K G, Gutiérrez M A and Hanssen R F 2014 Discontinuities without discontinuity: The weakly-enforced slip method; Comput. Meth. Appl. Mech. Eng. 271 144-166.

Volterra V 1907 Sur l'équilibre des corps élastiques multiplement connexes; In: Annales scientifiques de l'Ecole Normale superieure; Société mathématique de France, pp. 401-517. 